ORNL/TM-13074

Dist. Category UC-427

Fusion Energy Division

\title{
A LANDAU FLUID MODEL FOR DISSIPATIVE TRAPPED ELECTRON MODES
}

C. L. Hedrick

J.-N. Leboeuf

K. L. Sidikman

Date Published September, 1995

Prepared by

OAK RIDGE NATIONAL LABORATORY

Oak Ridge, Tennessee 37831

operated by

LOCKHEED MARTIN ENERGY SYSTEMS

for the

U.S. DEPARTMENT OF ENERGY

under Contract No. DE-AC05-84OR21400 


\section{DISCLAIMER}

This report was prepared as an account of work sponsored by an agency of the United States Government. Neither the United States Government nor any agency thereof, nor any of their employees, make any warranty, express or implied, or assumes any legal liability or responsibility for the accuracy, completeness, or usefulness of any information, apparatus, product, or process disclosed, or represents that its use would not infringe privately owned rights. Reference herein to any specific commercial product, process, or service by trade name, trademark, manufacturer, or otherwise does not necessarily constitute or imply its endorsement, recommendation, or favoring by the United States Government or any agency thereof. The views and opinions of authors expressed herein do not necessarily state or reflect those of the United States Government or any agency thereof. 


\section{DISCLAIMER}

Portions of this document may be illegible in electronic image products. Images are produced from the best available original document. 


\section{CONTENTS}

ACKNOWLEDGMENTS ............................................................................. v

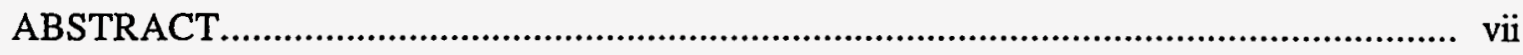

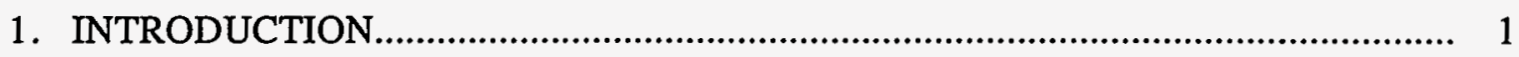

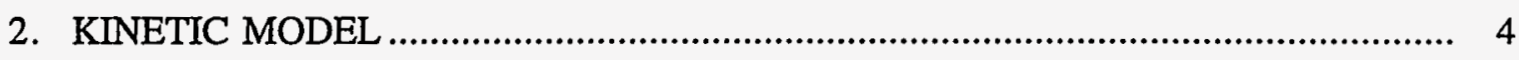

2.1. Kinetic Equation and Notation................................................................ 4

2.2. Local Dispersion Relation.......................................................................... 7

2.3. Sheared Magnetic Field ................................................................................. 8

2.4. Harmonic Oscillator Approximation.......................................................... 9

2.5. Kinetic Eigenvalues and Functions........................................................... 12

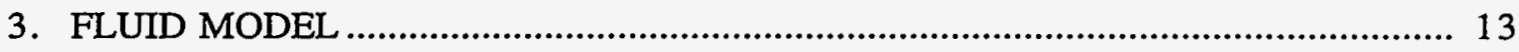

3.1. Evolution and Closure Equations ................................................................. 14

3.2. Fluid Response Function...................................................................... 16

3.3. Determination of Closure Coefficients ........................................................ 16

3.4. Linear Fluid Evolution Equations and Results ............................................ 20

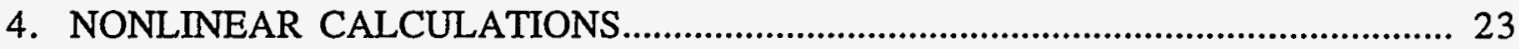

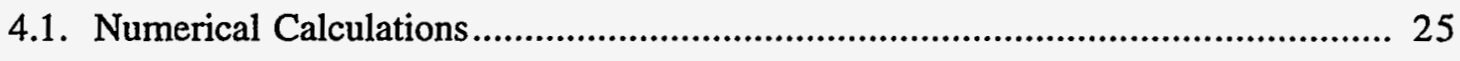

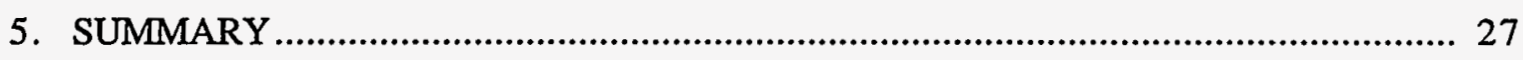

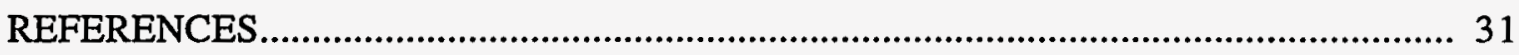

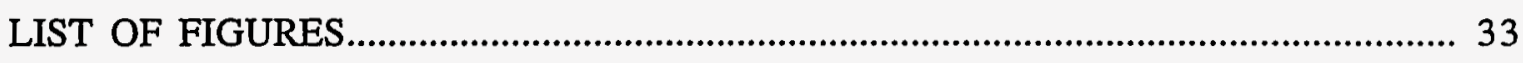




\section{ACKNOWLEDGMENTS}

The authors gratefully acknowledge the early work in this area by N. Dominguez. The authors would like to thank W. Dorland, G. Hammett, and J. Reynders for graciously providing us with their workstation version of the Linsker code, and R. D. Sydora for his CRAY version of the same. We would also like to acknowledge sage comments and advice on the part of R. D. Sydora and D. E. Newman throughout the course of this work. We are equally grateful to B. A. Carreras and R. A. Dory for their continued support and encouragements. This work is carried out as part of the U. S. Department of Energy High Performance Computing and Communications Initiative's Numerical Tokamak Grand Challenge Project. Computations performed during the course of this research were carried out at the National Energy Research Supercomputer Center (NERSC) and help from B. Curtis and R. Troutman is gratefully acknowledged for making the codes parallel on the CRAY YMP C90 and for visualization of the results. This work is supported by the U. S. Department of Energy under Contract No. DE-AC05-84OR21400 with Martin Marietta Energy Systems, Inc. 


\begin{abstract}
A Landau fluid model for dissipative trapped electron modes is developed which focuses on an improved description of the ion dynamics. The model is simple enough to allow nonlinear calculations with many harmonics for the times necessary to reach saturation. The model is motivated by a discussion that starts with the gyro-kinetic equation and emphasizes the importance of simultaneously including particular features of magnetic drift resonance, shear, and Landau effects. To ensure that these features are simultaneously incorporated in a Landau fluid model with only two evolution equations, a new approach to determining the closure coefficients is employed. The effect of this technique is to reduce the matching of fluid and kinetic responses to a single variable, rather than two, and to allow focusing on essential features of the fluctuations in question, rather than features that are only important for other types of fluctuations. Radially resolved nonlinear calculations of this model, advanced in time to reach saturation, are presented to partially illustrate its intended use. These calculations have a large number of poloidal and toroidal harmonics to represent the nonlinear dynamics in a converged steady state which includes cascading of energy to both short and long wavelengths.
\end{abstract}




\section{INTRODUCTION}

Here we discuss a gyro-Landau fluid ${ }^{1-11}$ model for Dissipative Trapped Electron Modes (DTEM).12-20 DTEM turbulence is one of the mechanisms invoked to explain fluctuations and transport at the core of tokamaks. ${ }^{12}$ To address core turbulence questions, fluid models have previously been used to perform spatially resolved calculations of DTEM turbulence. ${ }^{14,16}$ In contrast to some of the more abundant local descriptions ${ }^{15,17}$ where the evolution of the trapped electron species is followed in time, the spatially resolved models have largely concentrated on simplified trapped electron dynamics reduced to the " $i \delta$ model."13 These models have, so far, also been limited to highly collisional treatments of the ion dynamics in the absence of finite Larmor radius effects, which are less applicable to the hotter core plasmas of present-day tokamaks. In the gyro-Landau fluid model developed here, both of these restrictions on the ion dynamics are relaxed, and new effects caused by the velocity dependence of magnetic drifts are introduced.

Thus a major focus of this paper is on an improved description of the ion dynamics in a way which minimizes the number of evolution equations. The ultimate use of this model is in nonlinear calculations in which the dominant nonlinear effect is advection: the transport of fluctuating macroscopic quantities by the fluctuating $E \times B$ velocity. An example of such nonlinear calculations is included at the end of this paper.

The motivation for the simplified slab model developed here arises from the nonlinear effects it is intended to study. The nonlinearities cascade energy to both high and low perpendicular wave numbers. Thus there is little hope of reaching a saturated state unless the linearized modes are damped at high perpendicular wave numbers, and a sufficient number are included to dissipate the energy. Of course one can include ad hoc damping at high wave numbers, but the question then arises as to how sensitive the amplitude of the saturated state is to the ad hoc damping, and whether the magnitude of the ad hoc damping is credible (e.g., comparable to neoclassical diffusion in a tokamak). To answer these questions requires calculations with a large number of time steps for a substantial number of modes (the simple example discussed at the end of this paper has $10^{4}$ time steps and 192 poloidal mode numbers). Such calculations tax the memory available on present day computers and require considerable computation time. Since memory and time requirements increase at least linearly with the number of evolution equations, there is a premium placed on simplifying and minimizing the number of evolution equations.

To minimize the number of evolution equations we have introduced a technique which may be of use for other similar modes and problems. By making the closure coefficients functions of the poloidal mode number, we effectively reduce the matching of 
fluid and kinetic response functions from a two dimensional problem to one in a single variable. We focus on the features of DTEM that are important to retain and do not attempt to fit features of other modes, such as Ion Temperature Gradient (ITG) modes, $1,2,4,5,7,10,11$ or the electromagnetic Toroidal Alfvén Eigenfunctions (TAE).6,21,22,23,24 The advantage to this technique is that it reduces the number of evolution equations. The disadvantage is that one cannot reasonably expect that the closure coefficients chosen in this way will provide equally good fits to other types of fluctuations (e.g., ITG and TAE). This is in contrast to the approach taken in Refs. 19 and 20, which in principle can treat a variety of fluctuations, in toroidal geometry. At the time of this writing, the only published graphical comparison between their fluid treatment and linearized kinetic DTEM calculations as functions of perpendicular wave number is in Ref. 20. This comparison is for 9 or 10 evolution equations ( 6 ion evolution equations and 3 or 4 electron evolution equations) and is limited to positive growth rates (no marginally stable or damped cases are shown). Because of this, and because there are no published spectral decompositions of saturated nonlinear states, it remains to be seen how efficacious the approach taken in Refs. 19 and 20 will be for treating the problem examined in this paper.

To reiterate, we have focused on maintaining simplicity here and eschewed including effects that might be added at a later time, or are better treated in a more fully kinetic approach. In this regard we should mention that initial comparisons of this nonlinear fluid approach with one using gyrokinetic simulation techniques is extremely encouraging. ${ }^{25}$

While we use as simple a model as possible for the linear processes, we do include what seem to us to be the critical physical mechanisms. A key requirement is that the spectrum of linear modes should be stable (or damped) at high poloidal mode numbers to be able to legitimately use a finite number of modes. This requirement led us to include ion magnetic drift resonance damping, since otherwise, as we shall see, the modes are unstable for large poloidal mode number. 8,9 We also include ion Landau damping because it limits the radial extent of the unstable and moderately damped modes, and eliminates spurious radial oscillations. This also permits us to include shear damping, which significantly reduces the growth rate of the most unstable modes and helps suppress spurious noise. We also insist that the several macroscopic quantities have the correct parity with no discontinuities at the rational surface where the fluctuating electrostatic potential is largest. The above requirements allow us to determine coefficients in the closure relations in the Landau fluid approach.

There are a number of other approximations that we have made. One is for the electron linear response function, where we use a simple " $i \delta$-model" 13 appropriate for strongly dissipative trapped electrons. This approximation was replaced by a time evolution equation for the density and showed no differences at the linear level for the parameters of 
interest here, so we regard the " $i \delta$-model" as adequate for our present purposes. In this paper we include the ion curvature drift, but neglect the ion gradient-B drift. This reduces the amount of numerical computation by about two orders of magnitude at the linear level and can be compensated by modest changes in the numerical value of parameters (e.g., a $30 \%$ change in the $\delta$ parameter appearing in the electron model).

It was initially hoped that a combination of shear, Landau, and finite Larmor radius (FLR) effects (without ion magnetic drift resonance damping) would stabilize the modes at high poloidal mode number for the equilibrium parameters of interest. A sequence of increasingly sophisticated calculations convinced us that this was not the case and gave insights into which approximations were most accurate. While we do not wish to obscure the body of the paper with these matters, it does seem appropriate to mention them here in the introduction because they motivated our choices and may be useful in other applications.

The primary FLR effects come from the function $\Gamma_{0}(b) \equiv I_{0}(b) \exp (-b)$ in a simple slab model, where $b \equiv k_{\perp}^{2} \rho_{i}^{2}, k_{\perp}$ is the perpendicular wave number and $\rho_{i}$ is the ion gyroradius. In sheared geometry, the parallel wave number is a function of radial position through the equilibrium rotational transform or the "safety-factor." In sheared geometry b becomes a differential operator and does not commute with the parallel wave number. The numerical approach of Linsker ${ }^{26}$ avoids these problems. Although the Linsker approach is not especially well suited for nonlinear problems, it does allow one to compare approximations at the linear level with a more exact numerical computation.

Two simple approximations are available when dealing with more complex geometry. One is the Pade approximation, ${ }^{27,28} \Gamma_{0} \cong 1 /(1+b)$. The other is the Ross-Mahajan approximation, ${ }^{29,30}$ which we use in this paper. We compared numerical results using these two approximations with the approach of Linsker. For $b$ below ten, the Pade and RossMahajan approaches both gave reasonable approximations. For $|b|>10$, the Ross-Mahajan approach gave good agreement, while the Pade approximation did not.

We also examined the so-called Linsker effect $5,7,26$ for these modes (noncommutation of $\mathrm{b}$ and $k_{11}$ ). Analytically, one would expect this effect to be small because the equilibrium scale lengths are so much longer than the fluctuating quantity scale lengths. Numerically, we found that the effect was very small, so one could neglect the fact that the parallel wave number did not precisely commute with the differential operator $b$. However, we did note that it is possible to misinterpret numerical results and draw incorrect conclusions. The problem lies in the fact that different radial eigenmodes (e.g., "radial mode numbers" of zero and one) can have eigenvalues that are relatively close, and care must be taken to assure oneself of the continuity of the numerical results during a search for eigenvalues as a function of $b$. 
The paper is organized as follows: in Sec. II a kinetic model is developed from the gyro-kinetic equation. The properties of this kinetic model, which will be approximated by the fluid model, are developed and discussed. The effects of magnetic resonance damping, shear damping, and Landau damping are illustrated numerically and discussed analytically.

In Sec. III the fluid model is developed by taking moments of the gyro-kinetic equation and appending suitable closure relations. The coefficients in these closure relations are determined by constraints that allow matching of the fluid results to the kinetic results. The rationale for the choices of constraints is presented, particularly techniques that prevent spurious residual instabilities from occurring at the linear level. The linear fluid evolution equations are presented. The results of numerical comparison of the fluid and kinetic models are illustrated graphically.

In Sec. IV the nonlinear calculations are presented. The nonlinear fluid evolution equations, with advection, are presented, and the reason for an additional stabilizing term is discussed. The effect of this term is examined linearly, and toward the end of this section some nonlinear effects are shown. The numerical methods used for the nonlinear calculations are discussed. A particular nonlinear example is presented, which goes far beyond the transient phase and reaches nonlinear saturation.

In Sec. $\mathrm{V}$ we summarize and discuss the results of this paper, as well as some extensions.

\section{KINETIC MODEL}

In this section we develop the kinetic model. We also discuss the features of the kinetic model that are incorporated in the fluid model presented in Sec. III. We begin by introducing notation and developing expressions that will be needed in later sections. We focus first on simple slab geometry without shear and then introduce the changes needed to treat cases with sheared magnetic fields.

\subsection{Kinetic Equation and Notation}

Our starting point for ions is the ion Gyro-Kinetic Equation (GKE) for electrostatic fluctuations averaged over the gyro-angle:31,32

$$
\left(\omega-\omega_{d i}-k_{11} v_{\| 1}\right)\left\langle g e^{-i L}\right\rangle_{g y r o}=\frac{|e| \phi}{T_{i 0}}\left(\omega-\omega_{*_{i}}\right) J_{0}^{2} F,
$$

where the ion gyro-angle averaged first-order distribution function, $f_{1}$, is given by 


$$
f_{1}=\left\langle g e^{-i L}\right\rangle_{g y r o}-\frac{|e| \phi}{T_{i 0}}
$$

and the lowest order ion distribution function, F, has been assumed to be a Maxwellian.

From Eqs. (1) and (2) we find that the perturbed ion density is given by

$$
\frac{n_{i 1}}{n_{0}}=-\frac{|e| \phi}{T_{i 0}}\left(1+\frac{\omega_{*_{i}}-\omega}{\omega}\left\langle\frac{\omega J_{0}^{2} F}{\omega-\omega_{d i}-k_{11} v_{\|}}\right\rangle\right) .
$$

As mentioned in the introduction, here we only include the curvature drift and omit the gradient-B drift:

$$
\omega_{d i}=\frac{1}{2} \omega_{d 0} \frac{m_{i} v_{\|}^{2}}{T_{i 0}},
$$

and

$$
\omega_{d 0}=2 \frac{k_{\mathrm{y}} T_{i 0}}{|e| B R_{c}} .
$$

In this case, the velocity space average in Eq. (3) can be written as

$$
\left\langle\frac{\omega J_{0}^{2} F}{\omega-\omega_{d i}-k_{11} v_{\| 1}}\right\rangle=Q_{i} \Gamma_{0}\left(b_{i}\right),
$$

where

$$
\begin{aligned}
& \Gamma_{0}(b)=I_{0}(b) e^{-b}, \\
& b_{i}=k_{\perp}^{2} \rho_{i}^{2}=k_{\perp}^{2} \frac{T_{i 0}}{m_{i} \omega_{c i}^{2}}, \\
& Q_{i}=-\frac{1}{2} \lambda^{1 / 2} \frac{Z\left(\xi_{+}\right)+Z\left(\xi_{-}\right)}{\left(1+\frac{\lambda}{4 \zeta^{2}}\right)^{1 / 2}}, \\
& \xi_{ \pm}=\lambda^{1 / 2}\left(1+\frac{\lambda}{4 \zeta^{2}}\right)^{1 / 2} \pm \frac{\lambda}{2 \zeta},
\end{aligned}
$$




$$
\begin{aligned}
& \lambda=\frac{\omega}{\omega_{d 0}}, \\
& \zeta=\frac{\omega}{2^{1 / 2} k_{n} V_{T i}},
\end{aligned}
$$

and

$$
V_{T i}=\frac{T_{i 0}^{1 / 2}}{m_{i}^{1 / 2}}
$$

$\mathrm{Z}$ is the plasma dispersion function, ${ }^{33}$ and $I_{0}$ is a modified Bessel function.

If one can neglect ion magnetic drift, $\lambda \gg 1$, and $Q_{i}$ reduces to the relatively familiar expression

$$
Q_{i}=-\zeta Z(\zeta)
$$

In the limit of zero $k_{11}$ (infinite $\zeta$ ), Eq. (9) reduces to

$$
Q_{i}=-\lambda^{1 / 2} Z\left(\lambda^{1 / 2}\right)
$$

If the imaginary part of $\omega$ (and $\lambda$ ) is small compared to the real part, and $k_{\| 1}=0$, then

$$
\operatorname{Im}\left(Q_{i}\right)=-\pi^{1 / 2} \lambda^{1 / 2} \exp (-\lambda)=-\pi^{1 / 2} \frac{\omega^{1 / 2}}{\omega_{d 0}^{1 / 2}} \exp \left(-\omega / \omega_{d 0}\right)
$$

This behavior will dominate ion resonant damping.

Before considering the electron behavior, we note that just as one obtains the perturbed density from the GKE, one can obtain the fluctuating ion macroscopic parallel velocity:

$$
V_{\mathrm{ll}}=-\frac{|e| \phi}{T_{i 0}} \frac{\omega_{*_{i}}-\omega}{\omega}\left\langle\frac{\omega J_{0}^{2} F v_{\| 1}}{\omega-\omega_{d i}-k_{\mathrm{l}} v_{\mathrm{ll}}}\right\rangle
$$

From this we can see that $V_{11}$ is an odd function of $k_{11}$ [replace $k_{11}$ by $-k_{1 \mid}$ in Eq. (17), and then change the variable of integration from $v_{\| l}$ to $-v_{\|]}$. This is a specific example of the fact that odd moments of the perturbed distribution function are odd functions of $k_{\mathbb{l}}$, and even 
moments are even functions of $k_{\mathrm{fl}}$. We will make use of this fact in Sec. III when we need to impose constraints on the coefficients appearing in the closure relations.

For the electrons, following Carreras et al., ${ }^{16}$ we assume that we are in the dissipative trapped electron regime

$$
\frac{n_{e 1}}{n_{0}}=\frac{|e| \phi}{T_{e 0}}(1-i \delta),
$$

where

$$
\delta=1.5 \varepsilon^{3 / 2} \eta_{e} \omega_{*} / v_{e i}
$$

Note that here we have included electron temperature gradients, through $\eta_{e}$, while the ion treatment neglects ion temperature gradients. The electron temperature gradient is included so that neglect of frequency dependence in the electron response is more legitimate. Neglecting the ion temperature gradient is done for two reasons. One is that we do not want to complicate the analysis with the possibility of ion temperature gradient modes. The other reason is that if ion temperature gradients were carefully taken into account we would have to use at least three evolution equations in the Landau fluid treatment: ${ }^{6}$ by neglecting ion temperature gradients we only need to use two ion fluid evolution equations.

\subsection{Local Dispersion Relation}

To obtain the local dispersion relation, we substitute Eqs. (3) and (18) into the quasineutrality relation, $n_{e l}=n_{i 1}$. This is conveniently written in the form

$$
\frac{\omega}{\omega_{*_{e}}}=\frac{Q_{i} \Gamma_{0}}{1-i \delta+1 / \tau-Q_{i} \Gamma_{0} / \tau},
$$

where

$$
\tau=\frac{T_{i 0}}{T_{e 0}}
$$

This form of the dispersion relation given in Eq. (20) is useful for obtaining solutions by iteration. To obtain the initial guess for the iteration, one sets $Q_{i}=1$ and $\delta=0$ in the right side of Eq. (20):

$$
\frac{\omega}{\omega_{*_{e}}} \cong \frac{\Gamma_{0}}{1+\left(1-\Gamma_{0}\right) / \tau},
$$


which gives the generally correct trend that the real part of the frequency is near $\omega_{*_{e}}$ for small $b_{i}$ and decreases as $b_{i}$ increases. The most important use of Eq. (20) is for $k_{\| l}=0$, in which case $Q_{i}$ is given by Eq. (15). If $\omega_{d 0}$ is neglected, then Eq. (15) reduces to $Q_{i}=1$ and Eq. (20) becomes

$$
\frac{\omega}{\omega_{*_{e}}}=\frac{\Gamma_{0}}{1-i \delta+\left(1-\Gamma_{0}\right) / \tau},
$$

which clearly has a positive imaginary part, independent of $b_{i}$. This changes when nonzero $\omega_{d 0}$ is included.

Figure 1 illustrates some of the above points. From Fig. 1(a) we see that including nonzero $\omega_{d 0}$ has some quantitative effects on the real part of the frequency, $\omega_{r}$, but the general character is unchanged. On the other hand, we see from Fig. 1(b) that the growth rate, $\gamma$, is qualitatively changed by including nonzero $\omega_{d 0}$ : damping occurs at large poloidal mode number. Also note that there is a scale change between Figs 1(a) and 1(b), and that $\gamma<\omega_{r}$.

The qualitative change produced by nonzero $\omega_{d 0}$ is fairly simple to understand. Because $\omega_{*_{e}}$ and $\omega_{d 0}$ both increase with poloidal mode number, $\omega / \omega_{*_{e}}$ and $\omega / \omega_{d 0}$ both decrease with increasing poloidal mode number (monotonically). This causes the exponential associated with magnetic resonance damping in Eq. (16), $\exp \left(-\omega / \omega_{d 0}\right)$, to become larger with increasing poloidal mode number so that drift resonance damping is more effective at large poloidal mode number.

\subsection{Sheared Magnetic Field}

We now allow for sheared magnetic fields so that the parallel wave number varies in the radial, or $\mathrm{x}$-direction, as given below:

$$
k_{11}=k_{y} \frac{x}{L_{s}} .
$$

As discussed in the introduction, the shear length, $\mathrm{L}_{\mathrm{s}}$, is assumed so large that we can neglect spatial derivatives of the equilibrium quantities compared to derivatives of the fluctuating quantities. A consequence of this is that we can regard the differential operator,

$$
b_{i}=\rho_{i}^{2}\left(k_{y}^{2}-\frac{\partial^{2}}{\partial x^{2}}\right)=b_{y}-\frac{\partial^{2}}{\partial X^{2}},
$$


as commuting with $k_{\|}$. This allows us to obtain the differential equation for the electrostatic potential when shear is present by simply manipulating the local dispersion relation, Eq. (20). Thus,

$$
\Gamma_{0} \phi=\frac{\omega}{\left(\omega+\tau \omega_{*_{e}}\right) Q_{i}}[1+\tau(1-i \delta)] \phi
$$

To reduce Eq. (26) to a low-order differential equation we use the Ross-Mahajan approximation:29,30

$$
\Gamma_{0} \cong \Gamma_{0}\left(b_{y}\right)-\Gamma_{0}^{\prime}\left(b_{y}\right) \rho_{i}^{2} \frac{\partial^{2}}{\partial x^{2}}=\Gamma_{00}-\Gamma_{0}^{\prime} \frac{\partial^{2}}{\partial X^{2}}
$$

Then Eq. (26) becomes

$$
\frac{\partial^{2} \phi}{\partial X^{2}}-V \phi=0
$$

with the pseudo-potential, $V$, given by

$$
V=\frac{\Gamma_{00}-\frac{\omega}{\left(\omega+\tau \omega_{z_{e}}\right) Q_{i}}[1+\tau(1-i \delta)]}{\Gamma_{0}^{\prime}}
$$

Equation (28), together with the boundary conditions that $\phi$ vanish as $X$ tends to $\pm \infty$, can be solved numerically using standard shooting code techniques. However, it is also useful to approximate $V$ and solve Eq. (28) analytically.

\subsection{Harmonic Oscillator Approximation}

Here we expand the pseudo-potential given in Eq. (29) so that Eq. (28) reduces to the Schrodinger equation for the harmonic oscillator. The expansion formally assumes $x$ and $k_{11}$ are small. From Eq. (9) we obtain

$$
Q_{i} \cong Q_{0}+\frac{1}{2} k_{11}^{2} \frac{\partial^{2} Q_{i}}{\partial k_{11}^{2}}=Q_{0}+\frac{1}{2 \zeta^{2}} \frac{\partial^{2} Q_{i}}{\partial\left(\zeta^{-1}\right)^{2}}=Q_{0}+Q_{1} \frac{k_{1}^{2} V_{T i}^{2}}{\omega^{2}}
$$

with 


$$
Q_{0}=-\lambda^{1 / 2} Z\left(\lambda^{1 / 2}\right), \quad Q_{1}=-\frac{1}{4}\left[\lambda^{3 / 2} Z^{\prime \prime}\left(\lambda^{1 / 2}\right)+\lambda^{2} Z^{\prime}\left(\lambda^{1 / 2}\right)-\lambda^{3 / 2} Z\left(\lambda^{1 / 2}\right)\right]
$$

which allows us to write

$$
V \cong V_{0}+V_{1} X^{2}
$$

with

$$
V_{0}=\frac{1}{\Gamma_{0}^{\prime}}\left[\Gamma_{00}-\frac{\omega}{\left(\omega+\tau \omega_{*_{e}}\right)} \frac{\tau(1-i \delta)+1}{Q_{0}}\right]
$$

and

$$
V_{1}=\frac{1}{\Gamma_{0}^{\prime}} \frac{\omega}{\left(\omega+\tau \omega_{*_{e}}\right)} \frac{\tau(1-i \delta)+1}{Q_{0}} \frac{Q_{1}}{Q_{0}} \frac{\omega_{s}^{2}}{2 \omega^{2}},
$$

where

$$
\omega_{s} \equiv\left|\frac{k_{y} T_{i} c}{e B L_{s}}\right| .
$$

If we define $z$ by

$$
X=\alpha z
$$

with

$$
\alpha^{4}=\frac{1}{V_{1}}
$$

then Eq. (28) with Eq. (32) for $\mathrm{V}$ becomes

$$
\frac{\partial^{2} \phi}{\partial z^{2}}+\left(a-z^{2}\right) \phi=0
$$

with

$$
a=-\frac{V_{0}}{V_{1}^{1 / 2}}
$$

Equation (38) is of the same form as the Schrodinger equation for the harmonic oscillator. We demand solutions that vanish at $|x|=\infty$. This imposes the quantization condition

$$
a=2 p+1, \quad p=0,1,2,3 \ldots,
$$


and the solutions are

$$
\phi=H_{p}(z) \exp \left(-z^{2} / 2\right)
$$

Inserting Eq. (39) into Eq. (40) yields the radially resolved dispersion relation

$$
V_{0}=-V_{1}^{1 / 2}(2 p+1)
$$

Notice that implicit in this equation is a choice of branch (or sign) for the square root of $V_{1}$. The choice is governed by the fact that the perturbed electrostatic potential, and hence, $\exp \left(-z^{2} / 2\right)=\exp \left[-X^{2} /\left(2 \alpha^{2}\right)\right]=\exp \left(-V_{1}^{1 / 2} X^{2} / 2\right)$, should vanish as $|x|$ tends to infinity.

Thus,

$$
\operatorname{Re}\left(V_{1}^{1 / 2}\right)>0
$$

determines the branch of the square root.

Inserting Eqs. (33) and (34) into Eq. (42) yields the alternate form of the radially resolved dispersion relation

$$
\frac{\omega}{\omega_{*_{e}}}=\frac{Q_{e f f} \Gamma_{\infty 0}}{(1-i \delta+1 / \tau)-Q_{e f f} \Gamma_{00} / \tau},
$$

where

$$
Q_{e f f}=Q_{0}(1+\Delta)
$$

with

$$
\Delta=-(2 p+1)\left(-\frac{\Gamma_{0}^{\prime}}{\Gamma_{00}}\right) V_{1}^{1 / 2}
$$

Notice that Eq. (43) is of the same form as the local dispersion relation, Eq. (20). If $\Delta=0$, then Eqs. (43) and (44) reduce to the local dispersion relation for $k_{11}=0$. The iterative technique of solution discussed circa Eq. (20) also works well for Eq. (43).

The correction in the growth rate produced by nonzero $\Delta$ is due to shear stabilization. We can gain some insight into its origin by recalling that $V_{1}$ arises from nonzero $Q_{1}$. If we note that in the limit of infinite $\lambda, Q_{0}$ and $Q_{1}$ both tend to unity, we see from Eq. (14) that Eq. (30) in this limit simply corresponds to the leading terms of the asymptotic expansion 


$$
-\zeta Z(\zeta) \sim 1+\frac{1}{2 \zeta^{2}}=1+\frac{k_{11}^{2} V_{T i}^{2}}{\omega^{2}}
$$

As can be seen from Eqs. (30) or (46), $\Delta$ can also be viewed as originating from including ion acoustic or thermal corrections. Notice that Eq. (46) does not include the exponentially small term associated with Landau damping, $-i \pi^{1 / 2} \zeta \exp \left(-\zeta^{2}\right)$. As we shall see in the next section, Landau damping has important effects on the eigenfunctions, but not the eigenvalues.

\subsection{Kinetic Eigenvalues and Functions}

Here we present and discuss numerical results for the kinetic model. The focus is on motivating aspects of the kinetic model that are incorporated in the fluid model discussed in Sec. III. To do this we compare and contrast results from the three levels of approximation presented in the earlier part of this section.

Figure 2 shows the real and imaginary parts of the frequency vs. mode number for a set of parameters appropriate to the core of the TEXT tokamak.34,35 These parameters, which will be used throughout this paper for the numerical examples (with the exception of Fig. 3) are:

$$
\begin{array}{lll}
b_{i}=1.283 \times 10^{-4} \mathrm{~m}^{2} & \delta=1.364 \times 10^{-2} \mathrm{~m} & \tau=1 \\
\omega_{d 0} / \omega_{*_{e}}=1 / 3 & L_{s} / L_{n}=10.355 & \omega_{*_{e}} / \Omega_{i}=4.433 \times 10^{-5} \mathrm{~m}
\end{array}
$$

where $\Omega_{i}$ is the ion cyclotron frequency; we have chosen to characterize the perpendicular wave number in terms of the corresponding poloidal mode number, $\mathrm{m}$.

From Fig. 2(a), we see that the various levels of approximation all yield approximately the same results for the real part of the frequency. From Fig. 2(b) we see that the "full Z-function results," obtained with a shooting code using Eqs. (28) and (29), are quite close to those obtained from Eq. (43) for the harmonic oscillator approximation. Thus, the harmonic oscillator contains most of the information needed to obtain good approximations to the frequency and growth rate.

The primary difference between the harmonic oscillator and the local dispersion relation results is caused by inclusion of shear damping effects in the harmonic oscillator approximation. Figure 3(a) shows a case with less shear to illustrate this point. The parameters for the case illustrated in Fig. 3 are: 


$$
\begin{array}{lll}
b_{i}=7.160 \times 10^{-5} \mathrm{~m}^{2} & \delta=3.788 \times 10^{-3} \mathrm{~m} & \tau=0.588 \\
\omega_{d 0} / \omega_{*_{e}}=1 / 8 & L_{s} / L_{n}=25.874 & \omega_{*_{e}} / \Omega_{i}=4.433 \times 10^{-5} \mathrm{~m}
\end{array}
$$

At large poloidal mode number this difference is small, and comparison to Fig. 1(b) illustrates why it is necessary to include magnetic drift resonance damping effects. On the other hand, at small poloidal mode number, the stabilization is caused by shear damping. Accordingly, we see that it is important to retain both mechanisms.

Figure 3(b) shows eigenfunctions as functions of the radial variable, $x / \rho_{i}$, for the full Z-function and for the harmonic oscillator approximation. Notice in Fig. 3(b) that the harmonic oscillator approximation can give spurious oscillatory behavior extending to very large radial coordinate values. Landau fluid treatments of resistive g-modes 6 suggest that one can eliminate this spurious behavior by incorporating Landau damping. Anticipating the material discussed in the next section, Fig. 3(b) also shows that incorporating Landau effects in the fitting procedure eliminates the spurious oscillatory behavior.

Figures 2 and 3 are for the most unstable modes, which correspond to the radial quantum number $p=0$ in the harmonic oscillator approximation. Higher values of $p$ are stable for this set of equilibrium parameters (and for all other examples we have examined). The growth rate curves for these higher radial mode numbers are qualitatively similar to that for $p=0$, but are shifted downward, and the maximum is shifted to the left.

\section{FLUID MODEL}

In this section we develop a fluid model using techniques that are an extension of those previously employed for Landau fluids. To include magnetic drift resonance, the detailed approach taken is rather different from that employed in the past. ${ }^{6}$ Including magnetic drift necessitates increasing the number of closure relations and the number of closure coefficients to be determined. We have already seen another difference in Sec. II: the plasma response function, $Q_{i}$, is a function of two variables $\zeta$ and $\lambda$, whereas it is only a function of $\zeta$ if magnetic drift resonance is neglected. In the absence of magnetic drift, determining the closure coefficients could be reduced to fitting a ratio of polynomials involving the closure coefficients to the plasma dispersion function, $Z(\zeta)$. This fitting to $Z(\zeta)$ has some ambiguity: fits can be biased to be better at large or small values of $\zeta$. Accordingly, selecting a fit for $Z(\zeta)$ for any given instability should be done on a case by case basis (using, for example, solutions to the local dispersion relation to give guidance in the selection).

In the case at hand we again reduce the problem to fitting a function, $Q_{i}(\zeta, \lambda)$, by a 
ratio of polynomials. However, to fit the function adequately we make the closure coefficients functions of the poloidal mode number, rather than constants. Because the number of coefficients is proportional to the number of evolution equations, much better fits are allowed without having to increase the number of evolution equations.

Making the closure coefficients functions of poloidal mode number reduces the problem to having to fit $Q(\lambda, \zeta)$ only for the single variable $\zeta \equiv \omega /\left(\sqrt{2} k_{11} V_{T i}\right)$. The procedure described here allows one to assure oneself that the fit at small and modest $k_{\mathbb{l l}}$ (e.g., $\zeta \cong 1$ ) is in good agreement with the features of the kinetic eigenfunctions described in Sec. II. For example, we guarantee the effects associated with the harmonic oscillator approximation of Sec. II D, by insisting that the approximate fit match the two leading terms in

$$
Q(\lambda, \zeta)=Q(\lambda,)+k_{\mathbb{R}^{2}} \partial^{2} Q / \partial k_{\mathbb{R}} / 2+\cdots
$$

at finite $\omega_{d 0}$ or $\lambda$ (not just at zero or infinity ${ }^{10}$ ).

\subsection{Evolution and Closure Equations}

By taking moments of the GKE, Eq. (1), and using Eq. (2), we obtain the continuity and parallel momentum balance equations:

$$
\omega\left(n_{1}+n_{0} \frac{|e| \phi}{T_{i 0}}\right)-n_{0} k_{11} V_{11}-\omega_{d 0} \frac{n_{0}}{2}\left(\frac{T_{i 1}}{T_{i 0}}+\frac{n_{1}}{n_{0}}+\frac{|e| \phi}{T_{i 0}}\right)=n_{0}\left(\omega-\omega_{*_{i}}\right) \Gamma_{0} \frac{|e| \phi}{\mathrm{T}_{i o}}
$$

and

$$
\omega n_{0} V_{11}-k_{11} \frac{n_{0} T_{i 0}}{m_{i}}\left(\frac{T_{i 1}}{T_{i 0}}+\frac{n_{1}}{n_{0}}+\frac{|e| \phi}{T_{i 0}}\right)-\omega_{d 0}\left(\frac{q_{11}}{2 T_{i 0}}+\frac{3}{2} n_{0} V_{\| 1}\right)=0 .
$$

We close the system with the closure relations for the perturbed temperature and parallel heat flux. Note that if $\omega_{d 0}$ were zero, we would not need to include a closure relation for the parallel heat flux, $q_{\|}$:

$$
\frac{T_{i 1}}{T_{i 0}}=\left(2 a_{0}-1\right)\left(\frac{n_{1}}{n_{0}}+\frac{|e| \phi}{T_{i 0}}\right)+A_{1} 2^{1 / 2} \frac{V_{\| 1}}{V_{T i}}
$$

and

$$
\frac{q_{\| 1}}{2^{3 / 2} n_{0} V_{T i} T_{i 0}}=B_{0}\left(\frac{n_{1}}{n_{0}}+\frac{|e| \phi}{T_{i 0}}\right)+\left(b_{1}-\frac{3}{2}\right) \frac{V_{\|}}{2^{1 / 2} V_{T i}}
$$


$(L S)$

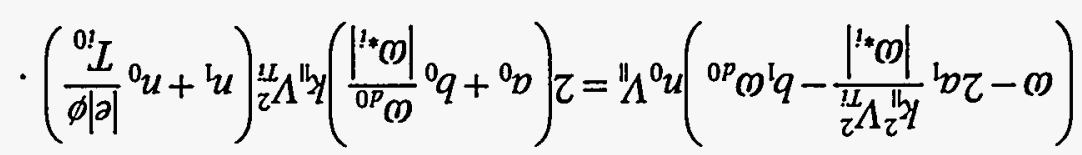

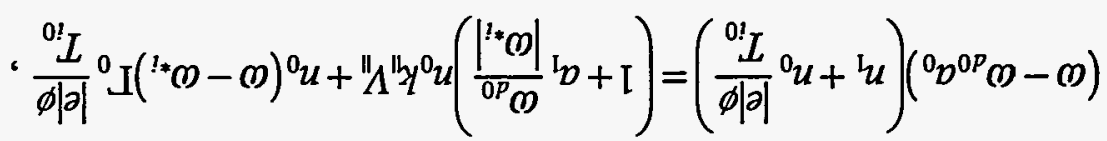

pue

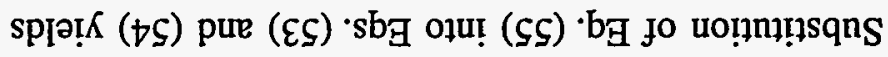

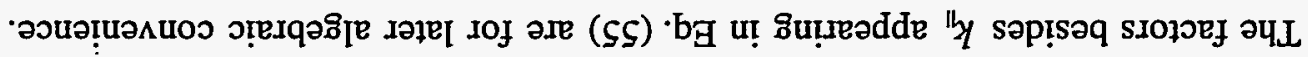

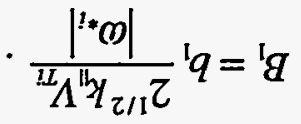

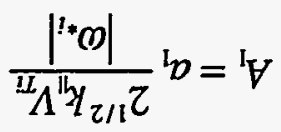

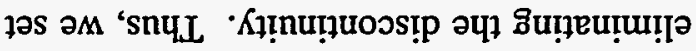

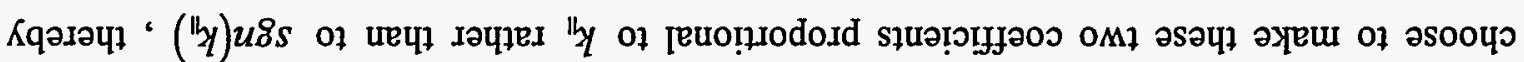

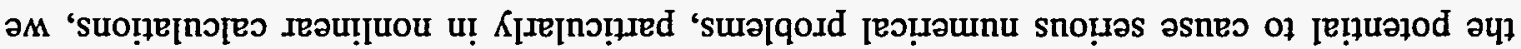

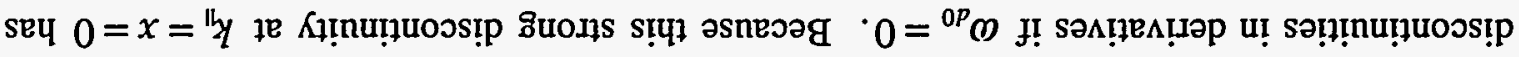

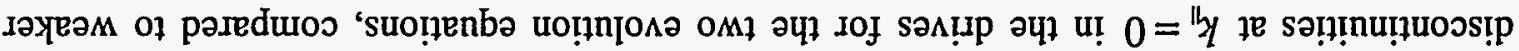

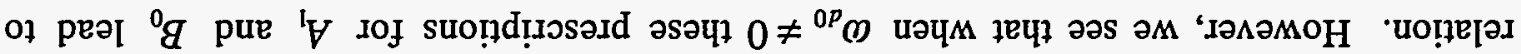

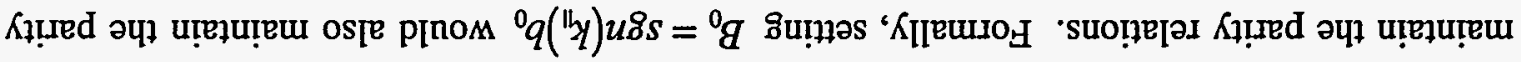

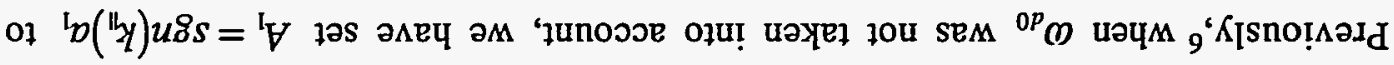

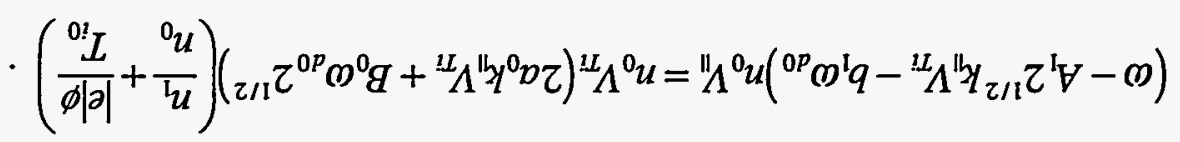

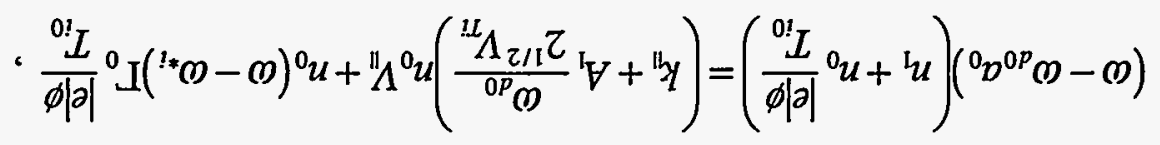

pue

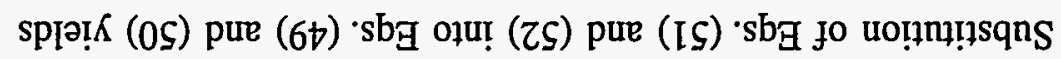

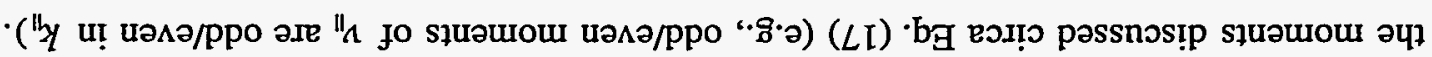

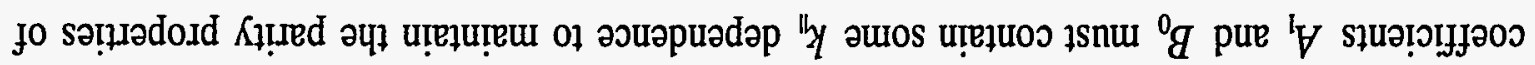

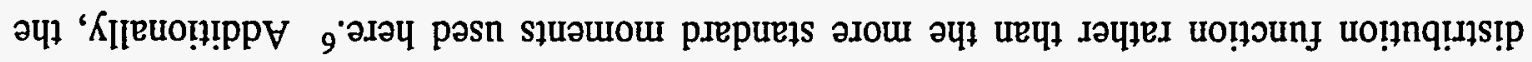

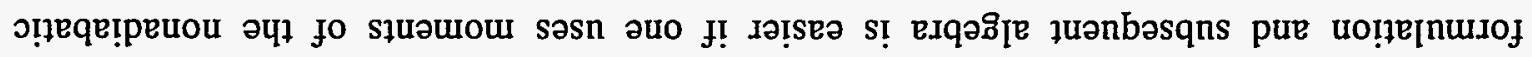

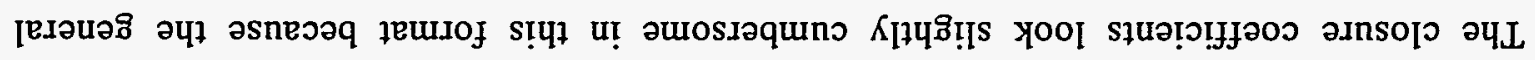




\subsection{Fluid Response Function}

In preparation for comparison of the kinetic and fluid response functions, we combine Eqs. (3) and (6) to obtain the kinetic result

$$
\frac{n_{1}}{n_{0}}=-\frac{|e| \phi}{T_{i 0}}\left(1+\frac{\omega_{*_{i}}-\omega}{\omega} Q_{i} \Gamma_{0}\right)
$$

To obtain the equivalent fluid result, we eliminate $V_{\| l}$ from Eqs. (56) and (57)

$$
\frac{n_{1}}{n_{0}}=-\frac{|e| \phi}{T_{i 0}}\left(1+\frac{\omega_{*_{i}}-\omega}{\omega} Q_{A} \Gamma_{0}\right),
$$

where

$$
\begin{aligned}
& Q_{A}=\frac{1-\frac{b_{1}}{\lambda}-\frac{a_{1}}{\zeta \zeta_{*}}}{1-\frac{a_{0}+b_{1}}{\lambda}+\frac{a_{0} b_{1}}{\lambda^{2}}-\frac{a_{0}}{\zeta^{2}}-\frac{a_{1}}{\zeta \zeta_{*}}-\frac{b_{0}}{\lambda \zeta \zeta_{*}}-\frac{a_{1} b_{0}}{\lambda^{2} \zeta_{*}^{2}}}, \\
& \zeta_{*}=\frac{\left|\omega_{* i}\right|}{2^{1 / 2} k_{11} V_{T i}},
\end{aligned}
$$

and $\lambda$ and $\zeta$ are given by Eqs. (11) and (12). Notice that if $Q_{A}$ were equal to $Q_{i}$, Eqs. (58) and (59) would be identical.

\subsection{Determination of Closure Coefficients}

We now determine the closure coefficients by applying various constraints so that we will have

$$
Q_{i} \cong Q_{A}
$$

We choose the constraints so that the most important features elucidated in Sec. II will be retained in the fluid approximation. The closure coefficients will be regarded as functions of the poloidal mode number to facilitate the fit associated with Eq. (62).

We first focus on fitting aspects of the most unstable modes which correspond to zero radial mode number in the harmonic oscillator approximation. We assume that the frequency of this mode is known from the kinetic model [e.g., from solving Eqs. (28) and (29) numerically with a shooting code]. We denote this frequency by $\omega_{E}$. 
Since we will first focus on small $k_{11}$, it is convenient to introduce

$$
\begin{aligned}
& y=\frac{1}{\zeta}=\frac{2^{1 / 2} k_{\|} V_{T}}{\omega} . \\
& a_{*}=\frac{\omega_{E}}{\omega_{*}} a_{1} \quad b_{*}=\frac{\omega_{E}}{\omega_{*}} b_{0} .
\end{aligned}
$$

With these definitions, Eq. (30) for the kinetic response function becomes

$$
Q_{i}=Q_{0}+\frac{1}{2} Q_{1} y^{2}+\ldots
$$

while Eq. (60) for the fluid response function becomes

$$
Q_{A}=\frac{1-\frac{b_{1}}{\lambda_{E}}-y^{2} a_{*}}{\left(1-\frac{b_{1}}{\lambda_{E}}\right)\left(1-\frac{a_{0}}{\lambda_{E}}\right)-y^{2}\left(a_{0}+a_{*}+\frac{a_{*} b_{*}}{\lambda_{E}^{2}}+\frac{b_{*}}{\lambda_{E}}\right)}
$$

Matching Eqs. (65) and (66) at $y=0$ leads to

$$
a_{0}=\lambda_{E} \frac{Q_{0}-1}{Q_{0}}
$$

We next insert Eq. (67) into Eq. (66) and expand as a power series in $y^{2}$ and then equate the coefficient of $y^{2}$ to that in Eq. (65), yielding

$$
Q_{0}\left(a_{0}+a_{*}+\frac{a_{*} b_{*}}{\lambda_{E^{2}}}+\frac{b_{*}}{\lambda_{E}}\right)-a_{*}=\frac{1}{2} \frac{Q_{1}}{Q_{0}}\left(1-\frac{b_{1}}{\lambda_{E}}\right) .
$$

Substitution of Eqs. (67) and (68) into Eq. (66) yieids

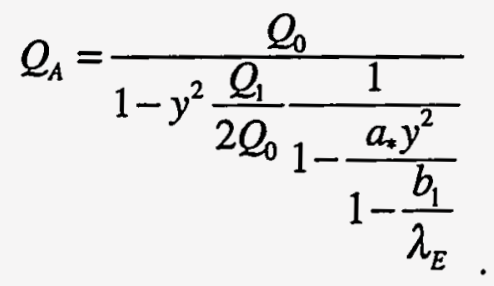


At this point we have matched the small $k_{1 l}$ features of $Q_{i}$ associated with the harmonic oscillator approximation of Sec. II, as can be seen by expanding Eq. (69) through $y^{2}$. This corresponds to matching the large $\zeta$ expansion of $Q_{i}$ through $\zeta^{-2}$.

We next impose a constraint that will assure us that Landau damping will be incorporated in the fluid approximation, so that the radial extent of the modes, etc. is limited by Landau effects. To do this we focus on a value of $\zeta$ of order unity, $\zeta_{F}$. In contrast to previous work, ${ }^{6}$ we do not focus on small values of $\zeta$ because this corresponds to very large $x$ and $k_{11}$ where the eigenfunctions are exponentially small, and hence of little importance. We define $Q_{F}$ to be the value of $Q_{i}$ at the specific intermediate value of $k_{1 \mid}$, with $\omega=\omega_{E}$. To match $Q_{A}$ to $Q_{i}$ at $\zeta_{F}$, it is convenient to introduce

$$
S_{F}=\zeta_{F}^{2}-\frac{Q_{1}}{2 Q_{0}} \frac{1}{1-\frac{Q_{0}}{Q_{F}}}
$$

If we set $y=1 / \zeta_{F}$ in Eq. (69) and insert this expression for $Q_{F}$ into Eq. (70) we obtain

$$
a_{*}=S_{F}\left(1-\frac{b_{1}}{\lambda_{E}}\right)
$$

For the results reported in this paper, the numerical value of $\zeta_{F}$ has been obtained from

$$
\zeta_{F}=\frac{\lambda_{E}^{1 / 2}}{2}
$$

which works well in practice. As can be seen from the square root appearing in Eqs. (9) and (10), this is the value where magnetic resonance and Landau effects are comparable.

At this point, if we allow for $\omega \neq \omega_{E}$, then

$$
Q_{A}=\frac{Q_{0}}{1+Q_{0}\left(\frac{\omega}{\omega_{E}}-1\right)-\frac{y^{2} \omega^{2}}{\omega_{E}^{2}} \frac{Q_{1}}{2 Q_{0}} \frac{1}{1+\left(\frac{\omega}{\omega_{E}}-1\right) \frac{S_{F}}{a_{*}}-S_{F} \frac{y^{2} \omega^{2}}{\omega_{E}^{2}}}} .
$$

If $\omega=\omega_{E}$, this reduces to 


$$
Q_{A}=\frac{Q_{0}}{1-y^{2} \frac{Q_{1}}{2 Q_{0}} \frac{1}{1-y^{2} S_{F}}}
$$

From Eq. (74) we see that we cannot develop any more relations for the closure coefficients if $\omega=\omega_{E}$. On the other hand, from Eq. (73) we see that $Q_{A}$ has a dependence on $a_{*}$ if $\omega \neq \omega_{\mathrm{E}}$, so we can develop a further relation to uniquely determine all the closure coefficients if we fit some aspect associated with modes other than the most unstable mode $(p=0)$.

To fit aspects of the less unstable modes, it is convenient to rewrite Eq. (60) as

$$
Q_{A}=\frac{1}{1-\frac{a_{0}}{\lambda}-\frac{\left(\frac{1}{\zeta}+\frac{a_{1}}{\lambda \zeta_{*}}\right)\left(\frac{a_{0}}{\zeta}+\frac{b_{0}}{\lambda \zeta_{*}}\right)}{1-\frac{b_{1}}{\lambda}-\frac{a_{1}}{\zeta \zeta_{*}}}}
$$

which simplifies the algebra for obtaining the asymptotic expansion

$$
Q_{A} \sim 1+\frac{a_{0}}{\lambda}+\left(\frac{1}{\zeta}+\frac{a_{1}}{\lambda \zeta_{*}}\right)\left(\frac{a_{0}}{\zeta}+\frac{b_{0}}{\lambda \zeta_{*}}\right)=1+\frac{a_{0}}{\lambda}+\frac{1}{\zeta^{2}}\left(1+\frac{a_{*}}{\lambda_{E}}\right)\left(a_{0}+\frac{b_{*}}{\lambda_{E}}\right)
$$

This is to be compared to the asymptotic expansion of the kinetic response function

$$
Q_{i} \sim 1+\frac{1}{2 \lambda}+\frac{1}{2 \zeta^{2}}
$$

We choose to match the $\zeta^{-2}$ terms in Eqs. (76) and (77). One reason for doing this is that Eq. (67) already gives an expression for $a_{0}$, so matching the $\lambda^{-1}$ terms is inappropriate. Indeed, the constraint leading to Eq. (67) is simply a better version of what would be obtained it we matched the $\lambda^{-1}$ terms in Eqs. (76) and (77) -- albeit for $\omega=\omega_{E}$. It is better in the sense that it incorporates magnetic drift resonance damping, which matching the $\lambda^{-1}$ terms in Eqs.(76) and (77) would not include. The terms proportional to $\zeta^{-2}$ in Eqs. (76) and (77) correspond to matching $Q_{1}$, but for $\omega \neq \omega_{E}$. This type of matching leads to shear damping through coupling to ion sound waves. Thus, it is hoped that by matching the $\zeta^{-2}$ terms in Eqs. (76) and (77), we will help damp any spurious noise that might occur in 
numerical calculations. Thus we obtain

$$
\left(1+\frac{a_{*}}{\lambda_{E}}\right)\left(a_{0}+\frac{b_{*}}{\lambda_{E}}\right)=\frac{1}{2}
$$

Equations (67), (68), (71), and (78) are sufficient to determine the closure coefficients. We obtain with the use of Eq. (64)

$$
\begin{aligned}
& a_{0}=\lambda_{E} \frac{Q_{0}-1}{Q_{0}}, \\
& a_{1}=\frac{\left|\omega_{*_{i}}\right|}{\omega_{E}} \frac{Q_{0}^{2} S_{F}}{Q_{1}}, \\
& b_{0}=\frac{\left|\omega_{*_{i}}\right|}{\omega_{E}}\left[\frac{\lambda_{E}^{2}}{2+\frac{2 a_{1} \omega_{E}}{\lambda_{E}\left|\omega_{*_{i}}\right|}}-\frac{\lambda_{E}\left(Q_{0}-1\right)}{Q_{0}}\right],
\end{aligned}
$$

and

$$
b_{1}=\lambda_{E}\left(1-\frac{Q_{0}^{2}}{Q_{1}}\right)
$$

\subsection{Linear Fluid Evolution Equations and Results}

From Eqs. (18), (27), (56), and (57) we obtain the fluid evolution equations

$$
\begin{gathered}
\left\{\left(\frac{\partial}{\partial t}-i \tau \omega_{*_{e}}\right)\left(1-\Gamma_{00}+\Gamma_{0}^{\prime} \rho_{i}^{2} \frac{\partial^{2}}{\partial x^{2}}\right)+\tau(1-i \delta)\left(\frac{\partial}{\partial t}+i a_{0} \omega_{d 0}\right)+i \tau \omega_{*_{e}}+i a_{0} \omega_{d 0}\right\} n_{0} \frac{|e| \phi}{T i} \\
=-i k_{11}\left(1+a_{1} \frac{\omega_{d 0}}{\left|\omega_{*_{i}}\right|}\right) n_{0} V_{\|}
\end{gathered}
$$

and

$$
\left(\frac{\partial}{\partial t}+i 2 a_{1} \frac{k_{11}^{2} V_{T i}^{2}}{\left|\omega_{*_{i}}\right|}+i b_{1} \omega_{d 0}\right) n_{0} V_{11}=-i 2 k_{11} V_{T i}^{2}\left(a_{0}+b_{0} \frac{\omega_{d 0}}{\left|\omega_{*_{i}}\right|}\right)[1+\tau(1-i \delta)] n_{0} \frac{|e| \phi}{T_{i}}
$$

The first of these two equations is essentially the ion density evolution equation, although it has been cast in a form that resembles the vorticity evolution equation of reduced 
magnetohydrodynamics (MHD). The second equation is, of course, the parallel momentum equation, or the parallel velocity evolution equation. We have chosen to emphasize the ion temperature in these equations, because ions are the focus for this paper. However, given the linear drive for instability that is provided by trapped electrons, many quantities are more naturally or compactly expressed in terms of the sound speed, $c_{s}{ }^{2}=T_{e} / m_{i}$, and the "sound" gyroradius, $\rho_{s}=c_{S} / \Omega_{i}$.

We have solved these equations numerically in the form of an initial value problem. The coefficients in Eqs. (80) and (81) were obtained from Eqs. (79), using $\omega_{E}$ determined from Eqs. (28) and (29) via a shooting code. Because this is a special case for the initial value code used to solve the nonlinear extensions of Eqs. (80) and (81), we will defer discussing some of these details until Sec. IV.

We have also solved Eqs. (80) and (81) numerically using a set of closure coefficients in which the condition relating to the more stable modes, Eq. (78), was replaced by the ad hoc condition $b_{0}=0$. With the latter set of coefficients, noise was observed to grow. With one proviso, using Eq. (79) cured this problem, indicating the utility of imposing the constraint associated with the more stable modes.

For very large poloidal mode number (e.g., 500), noise at large $x$ (or large $k_{11}$ ) was also observed to grow when using Eq. (79) for the coefficients. In an attempt to understand this phenomenon, the local dispersion relation was derived from Eqs. (80) and (81) -- a relatively simple quadratic equation in the frequency. At large $k_{11}$ one of the solutions was found to be

$$
\omega=2 a_{1} \frac{k_{1}^{2} V_{\mathrm{T} i}^{2}}{\left|\omega_{*_{i}}\right|},
$$

and the critical value of the poloidal mode number for the onset of the noise in the numerical calculations was observed to correspond to the imaginary part of $a_{1}$ becoming positive. We associate Eq. (82) with a residual ion acoustic mode which is poorly modeled at these high mode numbers.

In principle one could improve the fluid (and kinetic descriptions) of these ion acoustic modes so that they were stable in this extreme limit. However, there are an infinite number of such damped modes, ${ }^{36}$ so this task could well be very time consuming. Instead we have adopted the simpler approach of using coefficients calculated at $m_{\max }$ for all poloidal mode numbers above $m_{\max }$. One chooses $m_{\max }$ so that the imaginary part of $a_{1}$ is negative. For the results reported in this paper, $m_{\max }$ was chosen to occur near the local minimum in $\operatorname{Im}\left(a_{1}\right)$. 
Figure 4(a) compares two ways of obtaining the fluid model growth rates with the kinetic model results obtained using the full $Z$-function expression given by Eqs. (28) and (29). The first way used to determine the fluid model growth rates is to solve Eqs. (80) and (81) numerically as an initial value problem. The second way of obtaining the fluid model solutions is to replace $Q_{i}$ in Eq. (29) by $Q_{A}$ and solve the boundary-value problem with a shooting technique. Notice that the agreement between all three curves is good, except for poloidal mode number, $m$, above $m_{\max }=105$. For $m>m_{\max }$, the calculation of the coefficients was truncated as mentioned in the previous paragraph.

It will also be noted that there is some difference between the shooting and initial value numerical approaches to determining the fluid model growth rates at high poloidal mode number, $m$. We attribute this to the difficulty in using initial value techniques for damped modes. These damping rates are difficult to calculate accurately, since a decaying mode is strongly influenced by initial conditions and may never form a proper eigenfunction. To avoid this, we added a constant growth rate to the evolution equations for the damped modes to force an eigenfunction to grow. Then, the damping rate was obtained by subtracting this constant from the calculated growth rate. Figure 4(a) shows that this works well, until the modes are so heavily damped that the added growth rate strongly changes their eigenfunctions.

The closure coefficients used for the calculations shown in Fig. 4(a) were obtained using $\omega_{E}$ as determined from the full $Z$-function shooting code. Another more rapid, but more approximate, way to obtain $\omega_{E}$ for use in determining the closure coefficients is from the harmonic oscillator approximation dispersion relation, Eq. (43). Figure 4(b) shows a comparison of these two fits with the full $Z$-function results. Clearly both fits are good, but the fit using $\omega_{E}$ from the full $Z$-function shooting code is better. We will use this more accurate fit throughout the remainder of the paper.

Finally, a comparison of the full $Z$-function eigenfunctions, the harmonic oscillator approximation eigenfunctions, and those obtained using the fluid techniques of this section, are shown in Fig. 3. The comparison is for the equilibrium data set with the smaller shear effect (larger shear length). For the equilibrium with the larger shear effect used for the bulk of the paper (including the nonlinear calculations of the next section), the agreement between all three methods of calculating the eigenfunctions is so close that it is difficult to distinguish between them. 


\section{NONLINEAR CALCULATIONS}

Here we discuss nonlinear calculations using the fluid model. The nonlinear fluid evolution equations to be used are

$$
\begin{aligned}
\left\{\left(\frac{\partial}{\partial t}+\right.\right. & \left.\mathbf{V}_{E \times B} \cdot \nabla-i \tau \omega_{*_{e}}\right)\left(1-\Gamma_{00}+\Gamma_{0}^{\prime} \rho_{i}^{2} \frac{\partial^{2}}{\partial x^{2}}\right) \\
& \left.+\tau(1-i \delta)\left(\frac{\partial}{\partial t}+\mathrm{V}_{E \times B} \cdot \nabla+i a_{0} \omega_{d 0}\right)+i \tau \omega_{*_{e}}+i a_{0} \omega_{d 0}\right\} \frac{|e| \phi}{\mathrm{T}_{i}} \\
& =-i k_{1 \|}\left(1+a_{1} \frac{\omega_{d 0}}{\left|\omega_{*_{i}}\right|}\right) V_{\| 1}-v_{1} \rho_{i}^{4} \nabla_{\perp}^{4} \frac{|e| \phi}{\mathrm{T}_{i}}
\end{aligned}
$$

and

$$
\begin{aligned}
& \left(\frac{\partial}{\partial t}+\mathrm{V}_{E \times B} \cdot \nabla+i 2 a_{1} \frac{k_{1 \mid}^{2} V_{\mathrm{Ti}}^{2}}{\left|\omega_{*_{i}}\right|}+i b_{1} \omega_{d 0}\right) V_{\|} \\
& \quad=-i 2 k_{11} V_{\mathrm{Ti}}^{2}\left(a_{0}+b_{0} \frac{\omega_{d 0}}{\left|\omega_{*_{i}}\right|}\right)[1+\tau(1-i \delta)] \frac{|e| \phi}{\mathrm{T}_{i}}+v_{2} \rho_{i}^{2} \nabla_{\perp}^{2} V_{\| 1},
\end{aligned}
$$

where the fluctuation $E \times B$ velocity is given by

$$
\mathbf{V}_{E \times B}=c \frac{\mathbf{z x} \nabla \phi}{B}
$$

and the closure coefficients, $a_{0}, a_{1}, b_{0}, b_{1}$, are given by Eqs. (79) as explained in Sec. III.

There are two major differences between Eq. (83) and (84) and their linear precursors, Eqs. (80) and (81). The first difference is displayed on the left side of Eqs. (83) and (84) and represents addition of the advection terms:

$$
\frac{\partial}{\partial t} \rightarrow \frac{\partial}{\partial t}+\mathbf{V}_{E \times B} \cdot \nabla
$$

The terms upon which this operates can be associated with standard fluid terms as follows. If we use the small Larmor radius approximation $\Gamma_{0} \cong 1-b_{i}$, then

$$
\Gamma_{0}^{\prime} \rho_{i}^{2} \frac{\partial^{2}}{\partial x^{2}}-\Gamma_{00}+1 \cong-\rho_{i}^{2} \nabla_{\perp}^{2}
$$


and we associate $\nabla_{\perp}^{2} \phi$ with the vorticity (curl of the $E \times B$ velocity). This term is also associated with the polarization drift and is often so labeled in nonlinear contexts. The remaining term in Eq. (83) acted on by the operator given in Eq. (86) is proportional to $1-i \delta$ and arises from the fluctuating density in the electron " $i \delta$-model" given by Eq. (18). The advective term associated with the fluctuating density is often called the $E \times B$ nonlinearity for historical reasons. In Eq. (84) one sees a standard convective derivative operating on the parallel ion velocity.

The nonlinear terms discussed above lead to small scale length eddies, which can trigger numerical instabilities with scale lengths comparable to the radial grid spacing. This has led us to introduce the terms proportional to $v_{1}$ and $v_{2}$ in Eqs. (83) and (84). One motivation is that there certainly are such viscous effects occurring in any real plasma, and these effects tend to smooth the radial profiles. Another motivation is that such terms are known to stabilize this type of numerical instability. ${ }^{18}$ Although the viscosity coefficients $v_{1}$ and $v_{2}$ have been introduced as ad hoc adjustable parameters, we have found that levels comparable to neoclassical ones (100 to 200 times the perpendicular viscosity for collisional ions) produce numerical stability.

To determine the linear effect of adding the viscosity to the evolution equations, we have taken both analytic and numerical approaches. We have kept the distinction between the two coefficients, $v_{1}$ and $v_{2}$, to allow us to see which of the two evolution equations is most strongly affected by the viscosity. The analytic approach is carried out in two parts, the first of which is to analyze the local dispersion relation for $k_{\|}=0$. This leads to

$$
\frac{\omega}{\omega_{*_{e}}}=\frac{\tau \Gamma_{00}+a_{0} \frac{\omega_{d 0}}{\omega_{z_{e}}}(1+\tau(1-i \delta))-i \frac{\nu_{1}}{\omega_{*_{e}}} b_{y}^{2}}{1-\Gamma_{00}+\tau(1-i \delta)}
$$

from which one obtains the following approximate change in the growth rate produced by the viscosity:

$$
\gamma_{v i s c} \cong-\frac{v_{1} b_{y}^{2}}{1-\Gamma_{00}+\tau}
$$

This increase in damping is the dominant effect at large poloidal mode number where the viscosity terms have their largest impact.

At lower poloidal mode number there is a counterbalancing effect produced by a combination of viscosity, shear, and magnetic drift. From Eq. (83) we see that the viscous 
term changes the coefficient of $\partial^{2} / \partial x^{2}$ into $\left[\Gamma_{0}^{\prime}-2 i v_{1} b_{y} /\left(\omega+\tau \omega_{*_{e}}\right)\right] \rho_{i}^{2}$. When this change is traced through the harmonic oscillator approximation analysis of Sec. II, one finds that it leads to a real correction to the expression given for $\Delta$ in Eq. (45), which in turn increases the real part of the frequency through Eq. (43). The increase in the real part of the frequency reduces $\exp \left(-\omega / \omega_{d 0}\right)$, the dominant factor in the damping associated with $Q_{0}$. Thus, there can be a slight decrease in the damping at low poloidal mode number. This effect is seen in the numerical calculations discussed later, which involve time-advancing the linearized evolution equations: Eqs. (83) and (84) with the advection terms described in Eq. (86) switched off.

\subsection{Numerical Calculations}

We now briefly discuss the computer code used to obtain the linear and nonlinear results described in the remainder of this section. The three-dimensional (3-D) computer code DTEM/GyLa advances the Gyro-Landau fluid equations in time. It was developed from an existing code, DTEM, which was used to study the evolution of a single field. ${ }^{18}$ In that case, the system consisted of an evolution equation for the ion density and a single closure relation obtained from the parallel velocity evolution by assuming a large ion collision frequency. The time-advance was performed in a single step. The present code has two advantages over this earlier version. The accuracy and stability of the numerical scheme have been improved by the implementation of the two-step advance described in Ref. 18 . Also, the linear parallel damping produced by two evolution equations with Landau closure is much more realistic than ion collisional damping. However, even with the parallel damping well-represented in the present model, our experience with nonlinear calculations has been that numerical instabilities grow unless the perpendicular damping is enhanced [e.g., terms proportional to $v_{1}$ and $v_{2}$ in Eqs. (83) and (84)].

Like its predecessor in Ref. 18, the DTEM/GyLa code uses a sheared slab geometry. The straight slab is a local approximation to a torus with major radius $R_{0}$, centered on minor radius $r=r_{0}$. Within the slab, position is defined by rectangular coordinates related to the local toroidal directions: $x=r-r_{0}$ in the direction of equilibrium gradients, $y=r_{0} \theta$ in the direction of the poloidal angle $\theta$, and $z=R_{0} \zeta$ in the direction of the toroidal angle $\zeta$. (Here we use the standard symbol for this angle since no confusion should result from using the same symbol for the usual argument of the plasma dispersion function.) The $x$ coordinate covers a radial interval of size $a$. Limits on $y$ and $z$ are chosen to correspond to a full cycle of the angular coordinates.

The fluctuating fields are required to be periodic in the $y$ and $z$ directions, allowing 
them to be represented as discrete Fourier series in the corresponding periodic variables:

$$
\phi(x, \theta, \zeta)=\sum\left[\phi_{m, n}(x) \cos (m \theta+n \zeta)+\phi_{-m,-n}(x) \sin (m \theta+n \zeta)\right]
$$

for example. The poloidal and toroidal mode numbers $m$ and $n$ are related to the wave numbers, $m=k_{y} r_{0}$ and $n=k_{z} R_{0}$. All operations are performed in $(m, n)$-space, and the results are stored in spectral form. Convolutions are calculated directly. The $x$-dependence of the Fourier coefficients is represented on a discrete grid. At the edges of the computational box $(x= \pm a / 2)$, they are required to go to zero.

In general, numerical solutions of the linearized equations from the DTEM/GyLa code are in good agreement with those obtained analytically. Closure coefficients $a_{0}, a_{1}, b_{0}, b_{1}$ calculated from the prescription following Eq. (79) produce initial-value solutions in excellent agreement with boundary-value solutions obtained via the shooting method from the full kinetic, or full $Z$ model of Eq. (28). Figure 4 compares growth rates in the absence of viscosity, while Fig. 5 shows the eigenfunctions. When viscosity is introduced, there is a reduction in the growth rate that increases with poloidal mode number $m$, as shown in Fig. 6. This is in qualitative agreement with the local analytic result of Eq. (86), since $b_{y} \sim m^{2}$. The small effect at lower poloidal mode numbers due to interaction of viscosity, shear, and magnetic drift is also evident. In all the numerical cases shown, $v_{2}=v_{1}$.

The DTEM/GyLa code handles linear equations very well, but it was constructed primarily to perform nonlinear calculations with Eqs. (83) and (84). Although the code is fully 3-D, we have focused on 2-D (single-helicity) tests of the model. In these cases, the magnetic field was chosen so that the resonant surface for the $m / n=3 / 2$ helicity was located at $r=r_{0}$. Only Fourier modes of this helicity were retained, and a slab width of $a=120 \rho_{i}$ was used. Equilibrium quantities were specified by their magnitudes and scale lengths at $r=r_{0}$. These were held constant in the slab, except for the equilibrium magnetic field. The $x$-dependence of $k_{\| 1}$ given in Eq. (24) permits the "twist" of a field line to change, thus introducing magnetic shear into the model. (This $x$-variation also permits three-dimensional calculations with multiple rational surfaces, to be reported in a later paper.)

Quasilinear relaxation of equilibrium profiles was prohibited so that the steady state fluctuation level could be studied for a fixed set of equilibrium parameters. A steady state is expected when the number of Fourier components is large enough to provide an adequate energy sink, and the coupling to the energy source is sufficiently strong. For the current parameters, Fig. 6 shows that modes with $30<m<50$ are unstable. The maximum $m$ in the calculations was chosen to be 288 . This provides a large range of linearly stable modes that can act as an energy sink. Good coupling between the peak of the source $(m \sim 40)$ and the 
sink was guaranteed by keeping every component along the resonant helicity. The total number of Fourier components was then 192, counting both $(m, n)$ and $(-m,-n)$.

Numerical calculations with this mode selection, and 600 radial grid points, reached a steady state. All modes were initialized with equal amplitudes and random phases. After a transient, the root-mean-square (RMS) fluctuation levels at $r=r_{0}$ settled down around an average value, as shown in Fig. 7 for $v_{1} / \rho_{i} V_{\mathrm{T} i}=2.5 \times 10^{-4}$. A single-helicity calculation with this model requires a large number of Fourier components to produce converged spectra. In this case, Fig. 8 shows a moderate fall-off in the spectra at $r=r_{0}$ (about 1 decade) for $m>240$. However, for this viscosity, strong nonlinear interactions were confined to a very small range in $m$. As a result, the RMS fluctuation levels depend on the values of the sources and sinks since most of the energy remains in the driven and damped modes.

\section{SUMMARY}

In this paper, we have developed a Landau fluid model for dissipative trapped electron modes, which incorporates ion magnetic drift, Landau damping, and magnetic shear. We have indicated the motivation for choosing the closure relations needed for the Landau fluid model and exhibited a nonlinear calculation that was run to saturation.

While the linear model converges well, the various nonlinearities tend to cascade energy to higher wave numbers, creating eddies with very small radial scale lengths. To deal with these short scale length eddies, which are outside the scope of the original model, we have incorporated ad hoc viscosities.

For the nonlinear example quoted, the value of $v_{1} / \rho_{i} V_{\mathrm{T} i}$ corresponds to a neoclassical level of viscosity, about 180 times the perpendicular collisional viscosity for ions with the present parameters. Promising results have also been obtained by reducing $v_{1} / \rho_{i} V_{\mathrm{T} i}$ by a factor of 10. The fluctuation levels reach a steady state, and an increase in the Reynolds number indicates that strong nonlinear interactions now occupy a significant range in $m$. On the other hand, the spectra are not as well-converged, with only a factor of three fall-off at large $m$. To obtain the best features of both viscosity values, we have introduced a coefficient that varies with $m$. Thus, the viscosity is small at small-to-intermediate $m$, allowing for strong nonlinear interactions, and increases at large $m$, to ensure a converged spectrum. Calculations are being performed to investigate the effects of such a profile. In addition, a multiple helicity nonlinear calculation involving many more harmonics than the example cited in this paper has been performed and shows most of the same characteristics.

Comparisons of the fluid model developed in this paper with particle-in-cell gyrokinetic calculations are in progress. ${ }^{25}$ Linear and initial nonlinear calculations are 
encouraging. These calculations should serve to further elucidate the applicability of Landau fluid techniques. Since the two approaches employ quite different "coarse graining" (smoothing of short wavelength noise by ad hoc viscosity in the fluid case and by "finite particle size" in the gyro-kinetic approach) it is expected that the sensitivity of saturation amplitude, etc. to these effects will be explored.

Here we should mention that including gradient-B effects requires little modification of the formalism presented in this paper. A major difference is that instead of Eq. (6) one uses

$$
R(\lambda, \zeta, b) \equiv\left\langle\frac{\omega_{0}^{2} F}{\omega-\omega_{d i}-k_{11} v_{\|}}\right\rangle
$$

and the integral is no longer separable into $Q(\lambda, \zeta) \Gamma_{0}(b)$. The other major difference is that there are additional closure coefficients associated with the gradient- $\mathrm{B}$ drift. In principle, these coefficients might allow one to impose additional constraints. In practice, we have not found that the additional coefficients allow any particularly useful additional constraints for this type of mode. Accordingly, one can set these additional coefficients to zero and calculate $a_{0}, a_{1}, b_{0}$, and $b_{1}$ from Eqs. (30), (65), (70), and (79) with the simple replacement $Q \rightarrow R / \Gamma_{0}$. The nonlinear evolution equations, Eqs. (83) and (84), are unchanged, except for the fact that different numerical values of $a_{0}, a_{1}, b_{0}$, and $b_{1}$ are used.

Finally, we speculate on how the method described in this paper might be extended to treat toroidal geometry, because this is a topic of some interest, ${ }^{37}$ and because it illustrates the type of considerations necessary when applying or extending Landau fluid models. We note that the closure coefficients in this paper are relatively weak functions of the poloidal mode number, and that growth rates and frequencies are displayed as functions of the perpendicular wave number in Ref. 20. Taken together these observations suggest that one can again make the closure coefficients functions of the poloidal mode number, even though the eigenfunctions in toroidal geometry consist of a spectrum of poloidal harmonics.

For nonlinear computations, Dorland ${ }^{38}$ has advocated comparison of fluid and kinetic calculations at the linear level before being allowed to "sit at the table," and it is not very much more difficult to obtain the means for calculating the kinetic results before fitting than after. Thus, suppose that one has a means for numerically calculating the linearized kinetic solutions. By using these numerical solutions one can calculate the analogs of the nonadiabatic response functions $Q_{0}, Q_{1}$ and $Q_{F}$ in Sec. III, and calculate quantities such as $\mathbf{v}_{d} . \nabla \phi$ which is the analog of $\omega_{d 0} \phi$ appearing in Eqs. (56) and (57). This would allow one to impose the analogs of the three constraints involving the most unstable modes given in Sec. 
III. Whether it is better to use a single poloidal harmonic in performing these calculations or to use averages over the harmonics constituting the eigenfunction in question can only be determined by trial and error. For the less unstable modes it may be sufficient to match the approximate non-adiabatic response function to $\Gamma_{0}\left[1+1 /\left(2 \zeta^{2}\right)\right]$ for large $\zeta$ and $\lambda$, as was done in this paper. The four constraints indicated are sufficient to determine the four closure coefficients as in this paper. We repeat that these ideas are only a starting point and one should anticipate having to deal with unforeseen difficulties.

While it is probably not a problem in fitting coefficients, the fact that the magnetic drift varies with poloidal angle in toroidal geometry should lead to some differences. For the slab model discussed in this paper it has been tacitly assumed that the average of $\omega_{*} \omega_{d}$ over a flux surface was negative (otherwise ideal MHD interchanges would be unstable), which leads to the stabilizing resonance between $\omega$ and $\omega_{d i}$. In toroidal geometry this stabilizing resonance will not occur in the bad curvature region, so one might expect that there will be a tendency for the mode to concentrate in the bad curvature region. However, such concentration will lead to variation along the field lines that will lead to increased Landau damping, which will limit the concentrating of the mode in the bad curvature region. A crude assessment of these competing effects suggests that for some equilibrium parameters the damping of higher poloidal mode numbers could be reduced and the marginal stability point moved to higher poloidal mode number. A consequence of this is that it would be necessary to retain more poloidal harmonics than would otherwise be the case. Clearly one would want to have a more accurate assessment of this possibility before entering into a calculation that might be beyond the scope of near term computing resources. 


\section{REFERENCES}

1. G. W. Hammett and F. W. Perkins, Phys. Rev. Lett. 64, 3019 (1990).

2. W. Dorland, G. W. Hammett, Liu Chen, W. Park, S. C. Cowley, S. Hamaguchi, and W. Horton, Bull. Am. Phys. Soc. 35, 2005 (1990) (Paper 4R10).

3. C. L. Hedrick and J.-N. Leboeuf, Bull. Am. Phys. Soc. 35, 2005 (1990) (Paper 4R13).

4. G. W. Hammett, Bull. Am. Phys. Soc. 36, 2454 (1991) (Paper 8I3).

5. G. W. Hammett, W. Dorland, and F. W. Perkins, Phys. Fluids B 4, 2052 (1992).

6. C. L. Hedrick and J.-N. Leboeuf, Phys. Fluids B 4, 3915 (1992).

7. W. Dorland and G. W. Hammett, Phys. Fluids B 5, 812 (1993).

8. C. L. Hedrick, J.-N. Leboeuf, and K. L. Sidikman, Bull. Am. Phys. Soc. 38, 2101 (1993) (Paper 9P13).

9. K. L. Sidikman, C. L. Hedrick, and J.-N. Leboeuf, Bull. Am. Phys. Soc. 38, 2101 (1993) (Paper 9P14).

10. R. E. Waltz, R. R. Dominguez, and G. W. Hammett, Phys. Fluids B 4, 3138 (1992).

11. R. E. Waltz, G. D. Kerbel, and J. Milovich, Phys. Plasmas 1, 2229 (1994).

12. B. B. Kadomtsev and O. P. Pogutse, Sov. Phys. JETP 24, 1172 (1967).

13. P. W. Terry and W. Horton, Phys. Fluids 25, 491 (1982).

14. R. E. Waltz, Phys. Fluids B 2, 2118 (1990).

15. O. T. Kingsbury and R. E. Waltz, Phys. Fluids B 3, 3539 (1991).

16. B. A. Carreras, K. Sidikman, P. H. Diamond, P. W. Terry, and L. Garcia, Phys. Fluids B 4, 3115 (1992).

17. D. E. Newman, P. W. Terry, P. H. Diamond, Y. M. Liang, G. G. Craddock, A. E. Konoges, and J. A. Crotinger, Phys. Plasmas 1, 1592 (1994), and references cited therein.

18. K. L. Sidikman, B. A. Carreras, and L. Garcia, J. Comput. Phys. 114, 100 (1994).

19. G. W. Hammett, M. A. Beer, J. C. Cummings, W. Dorland, W. W. Lee, H. E. Mynicks, S. E. Parker, R. A. Santoro, M. Artun, H. P. Furth, T. S. Hahm, G. Rewoldt, W. M. Tang, R. E. Waltz, G. D. Kerbel, and J. Milovich, Advances in Simulating Tokamak Turbulent Transport, in Proceedings of the 15th International Conference on Plasma Physics and Controlled Fusion Research, Seville, Spain 1994 (to be published by the International Atomic Energy Agency, Vienna, 1995) Paper No. IAEA-CN-60/D-2-II-1.

20. Michael Alan Beer, Gyrofluid Models of Turbulent Transport in Tokamaks, Ph.D. Dissertation, Princeton University, 1995 (copyright 1994).

21. D. A. Spong, B. A. Carreras, and C. L. Hedrick, Phys. Fluids B 4, 3316 (1992).

22. D. A. Spong, B. A. Carreras, C. L. Hedrick, N. Dominguez, L. A. Charlton, P. J. Christenson, and J-N. Leboeuf, Physica Scripta 45, 159 (1992).

23. D. A. Spong, B. A. Carreras, and C. L. Hedrick, Phys. Plasmas 1, 1503 (1994).

24. D. A. Spong, B. A. Carreras, C. L. Hedrick, and J-N. Leboeuf, Energetic Particle Destabilization of Shear Alfvén Waves in Stellarators and Tokamaks, in Proceedings of the 15th International Conference on Plasma Physics and Controlled Fusion Research, Seville, Spain 1994 (to be published by the International Atomic Energy Agency, Vienna, 1995) Paper No. IAEA-CN-60/D-2-II-3.

25. R. Sydora, private communication, April 5, 1995.

26. R. Linsker, Phys. Fluids 24, 1485 (1981)

27. C.-T. Hsu, Ph.D. dissertation, The University of Texas at Austin, 1986.

28. C. T. Hsu , R. D. Hazeltine, and P. J. Morrison, Bull. Am. Phys. Soc. 30, 1461 (1985).

29. D. W. Ross and S. M. Mahajan, Phys. Rev. Lett. 40, 324 (1978).

30. D. W. Ross and S. M. Mahajan, Phys. Fluids 22, 294 (1979).

31. P. J. Catto, W. M. Tang, and D. E. Baldwin, Plasma Phys. 23, 639 (1981).

32. C. L. Hedrick, J.-N. Leboeuf, and D. A. Spong, Phys. Fluids B 4, 3869 (1992).

33. B. D. Fried and S. D. Conte, The Plasma Dispersion Function (Academic Press, New York, 1961) pp. 1-8.

34. D. W. Ross, P. M. Schoch, J. W. Heard, T. P. Crowley, and R. L. Hickock, Nucl. Fusion 31, 1355 (1991).

35. A. J. Wooton, M. E. Austin, R. D. Bengtson, J. A. Boedo, R. V. Bravenec, D. L. Brower, J. 
Y. Chen, G. Cima, P. H. Diamond, R. D. Durst, P. H. Edmonds, S. P. Fan, M. S. Foster, J. C. Forster, R. Gandy, K. W. Gentle, R. L. Hickock, Y. X. Hey, S. K. Kim, H. Lin, N. C. Luhmann, S. C. McCool, W. H. Miner, A. Ouroura, D. M. Patterson, W. A. Peeples, P. E. Phillips, B. Richards, Ch. P. Ritz, T. L. Rhodes, D. W. Ross, W. L. Rowan, P. M. Schoch, D. Sing, E. J. Synakowski, P. W. Terry, K. W. Wenzel, J. C. Wiley, X. Z. Yang, X. H. Yu, Z. Zhang, S. B. Zheng, Plasma Phys. and Controlled Fusion 30, 1479 (1988).

36. Burton D. Fried and Roy W. Gould, Phys. Fluids 4, 139 (1961).

37. G. W. Hammett, private communication, April 6, 1995

38. W. Dorland, private communication, April 5, 1995. 


\section{Figure Captions}

Fig. 1 Real frequency (a) and growth rate (b) versus poloidal mode number, $m$, from the local dispersion relation for $k_{11}=0$. The solid curves include magnetic drift effects, while the dashed curves omit them. The important effect is in the growth rate, which is stabilized at high $m$ by magnetic drift. The parameters for this example are given in Eq. (47).

Fig. 2 Real frequency (a) and growth rate (b) versus poloidal mode number, $m$. The solid curves are for the full plasma dispersion function, $Z$. The open circles are for the harmonic oscillator approximation, and are quite close to the full $Z$ results. For reference, we show the local dispersion results of Fig. 1 with the dashed curves.

Fig. 3 Growth rates (a), and eigenfunctions for $m=15(b)$ for the parameter set given by Eq. (48). The solid curves are for the full $Z$ results. The open circles are for the harmonic oscillator results. In Fig. 3(a) the dashed line is for the local dispersion relation, as in Fig. (2), which has stronger shear effects. In Fig. 3(b), the dashed curves are for the eigenfunctions associated with the Landau approximation, discussed in Sec. III.

Fig. 4 Growth rates versus $\mathrm{m}$. The full $Z$ results are shown with open diamonds in Figs. 4(a) and (b). A comparison of shooting and initial value code results for the Landau fluid with coefficients obtained from the full $Z$ expression for $\omega_{E}$ is shown in Fig. 4(a). Figure 4(b) shows a comparison of the fit of Fig. 4(a), with that for a set of Landau closure coefficients obtained using the harmonic oscillator approximation for $\omega_{E}$.

Fig. 5 Eigenfunctions versus the radial coordinate for the parameters given in Eq. (47) for $m=15$. The initial value and shooting codes for the Landau fluid model both produce eigenfunctions that are virtually indistinguishable from the full $Z$ eigenfunction.

Fig. 6 Growth rate versus poloidal mode number, $m$. The open diamonds are for zero viscosity, while the solid circles are for $v_{1} / \rho_{i} V_{\mathrm{T} i}=2.5 \times 10^{-4}$. The effect of finite viscosity is in reasonable agreement with the analytic treatment given in the text.

Fig. 7 Fluctuating electrostatic potential (solid) and parallel velocity (dashed) magnitudes versus time for the parameters given in Eq. (47) and $v_{1} / \rho_{i} V_{T i}=2.5 \times 10^{-4}$. The calculation goes well past the initial transient $\left(\Omega_{i} t<2 \times 10^{4}\right)$ and into an approximate saturated state.

Fig. 8 Fluctuating potential (solid) and parallel velocity (dashed) square magnitudes versus poloidal mode number at the rational surface averaged over time $\left(6.0 \times 10^{4} \leq \Omega_{i} t \leq 1.0 \times 10^{5}\right)$. 


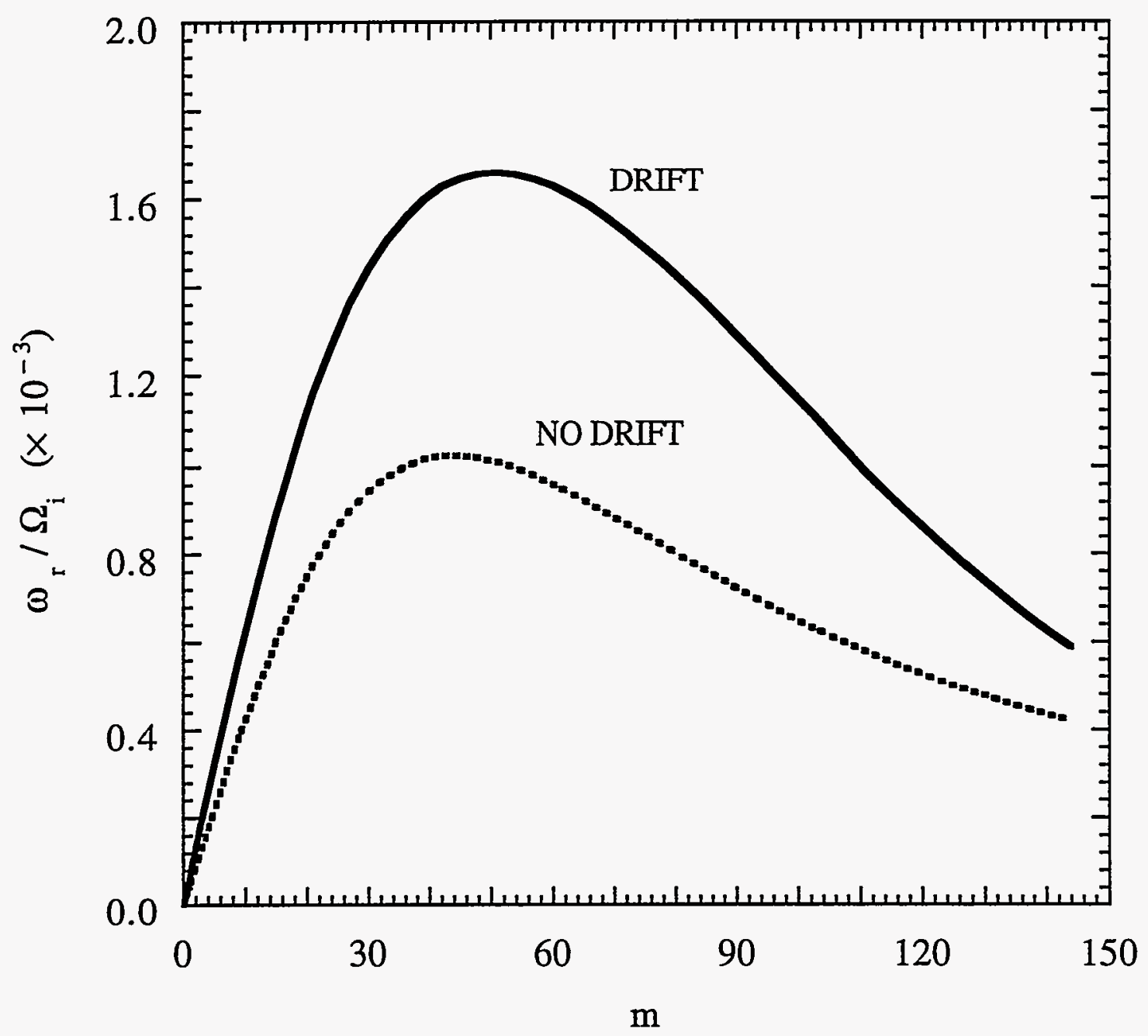

FIGURE 1a 


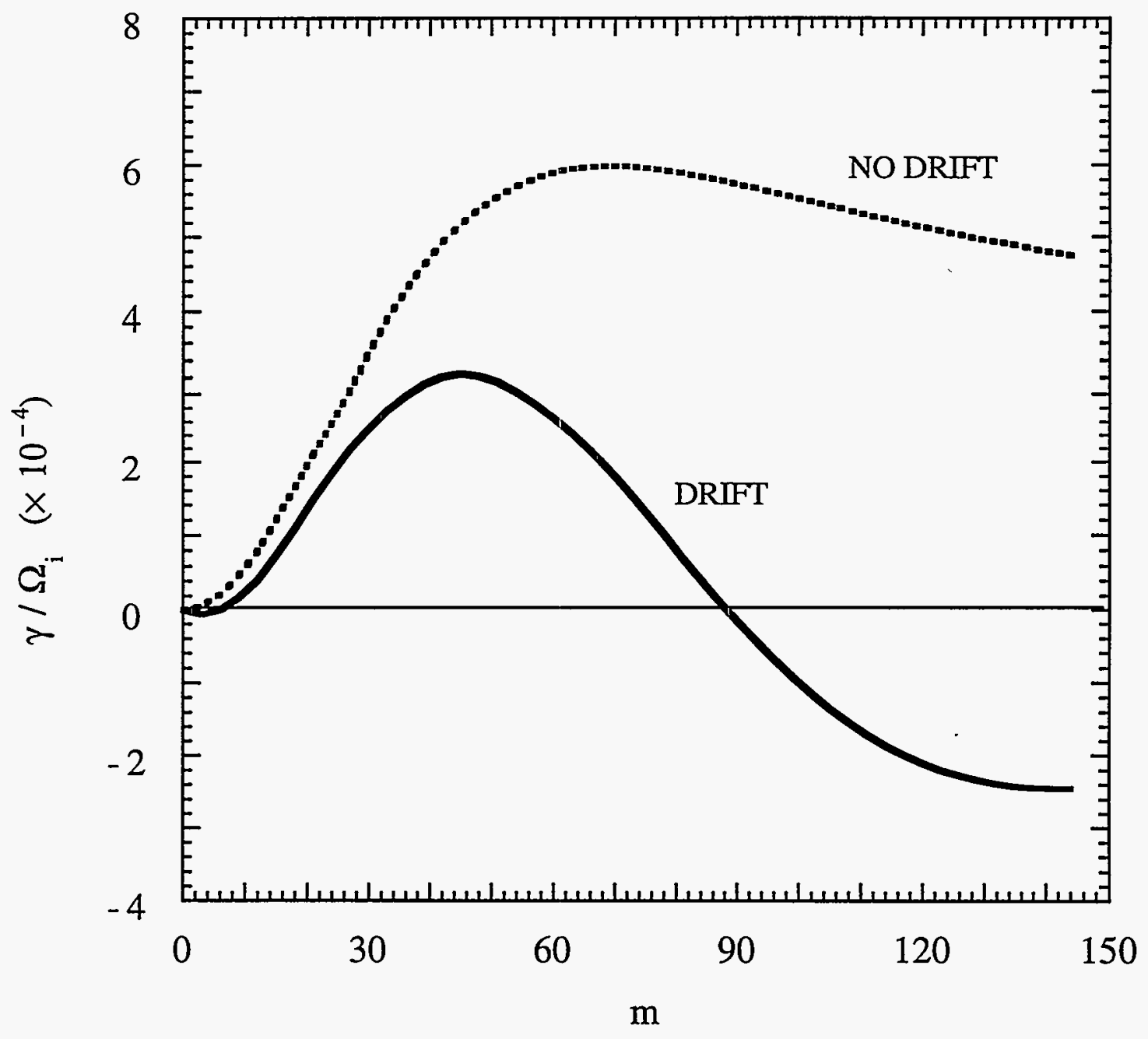

FIGURE 1b 


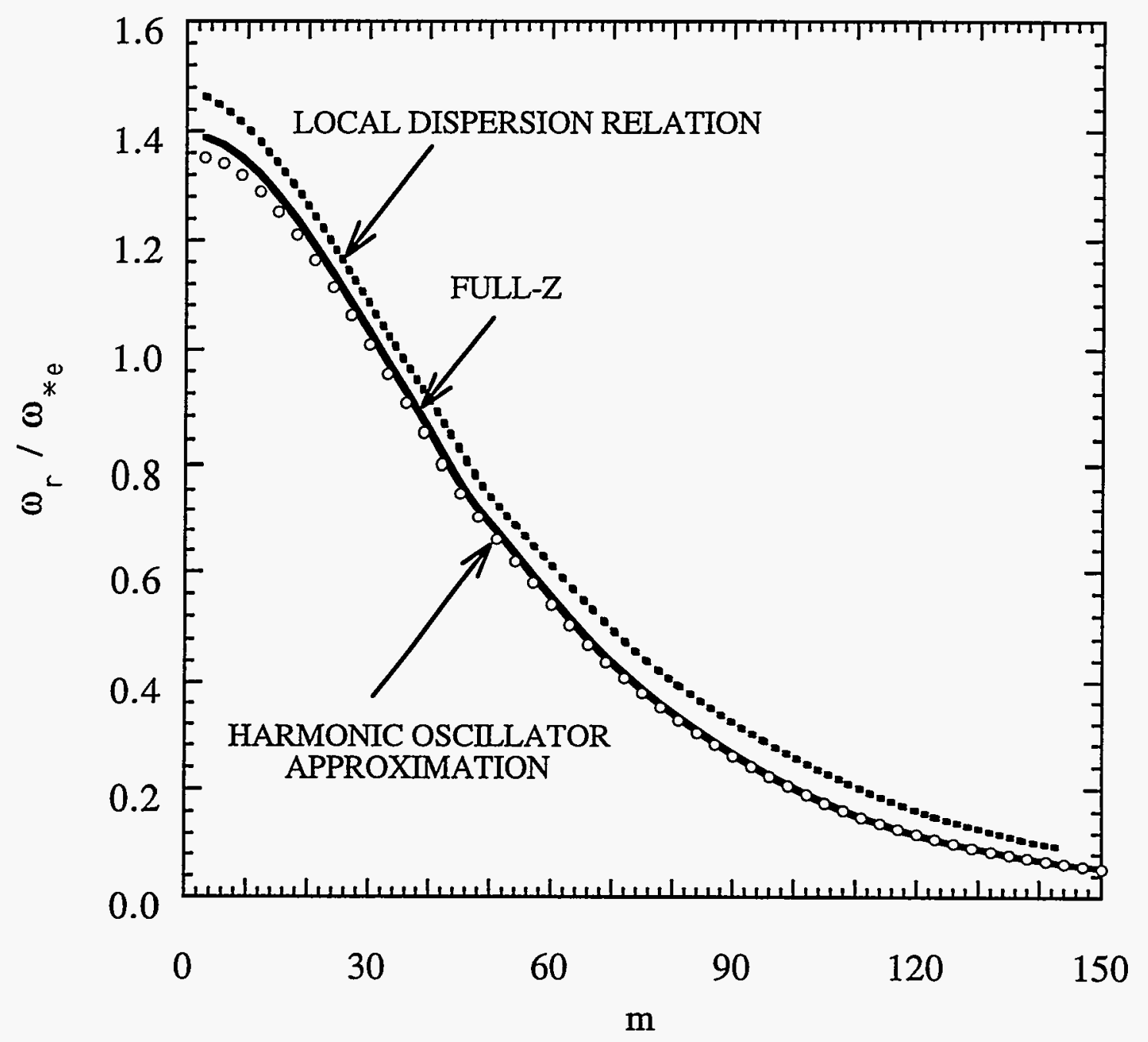

FIGURE 2a 


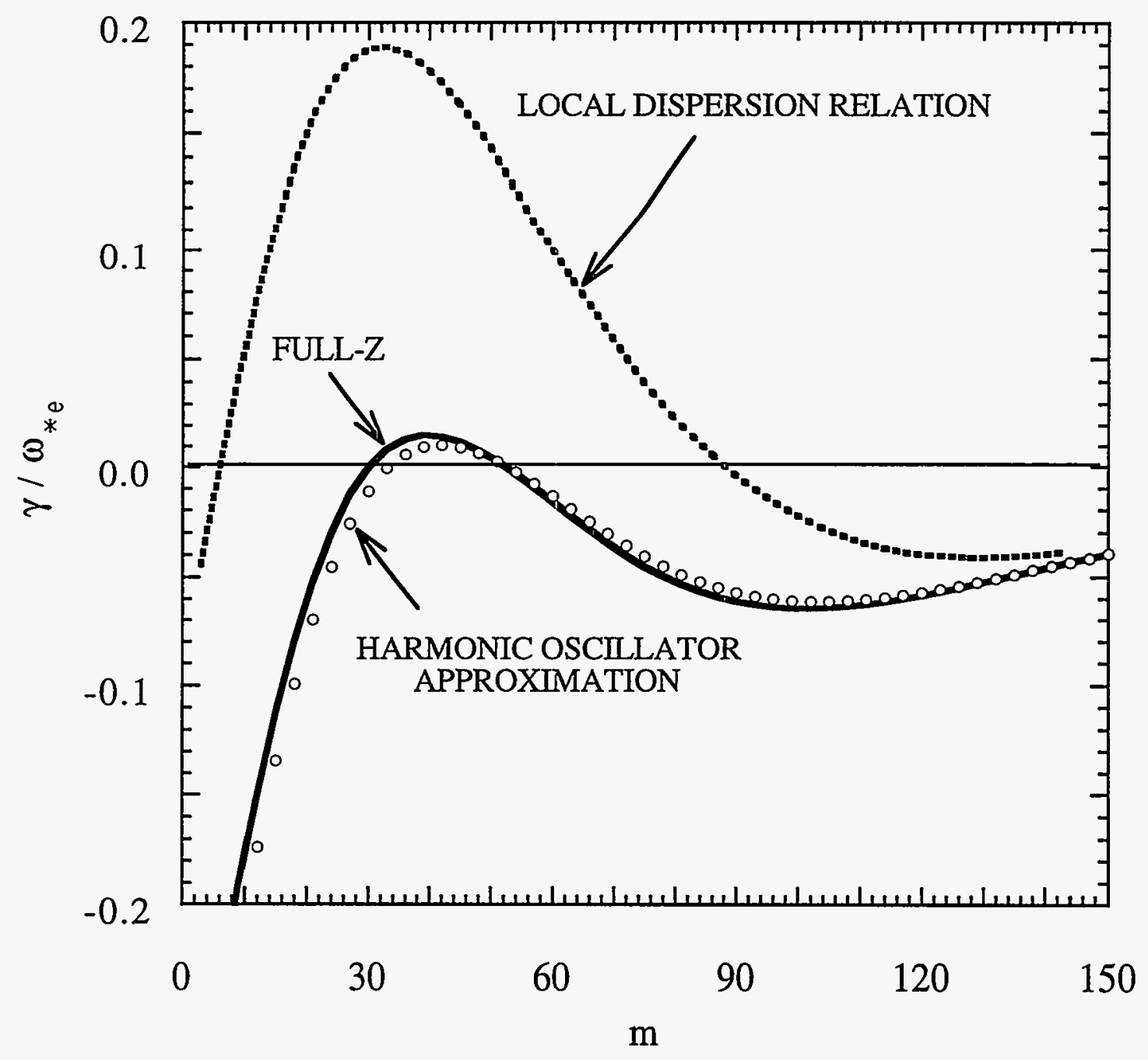

FIGURE 2b 


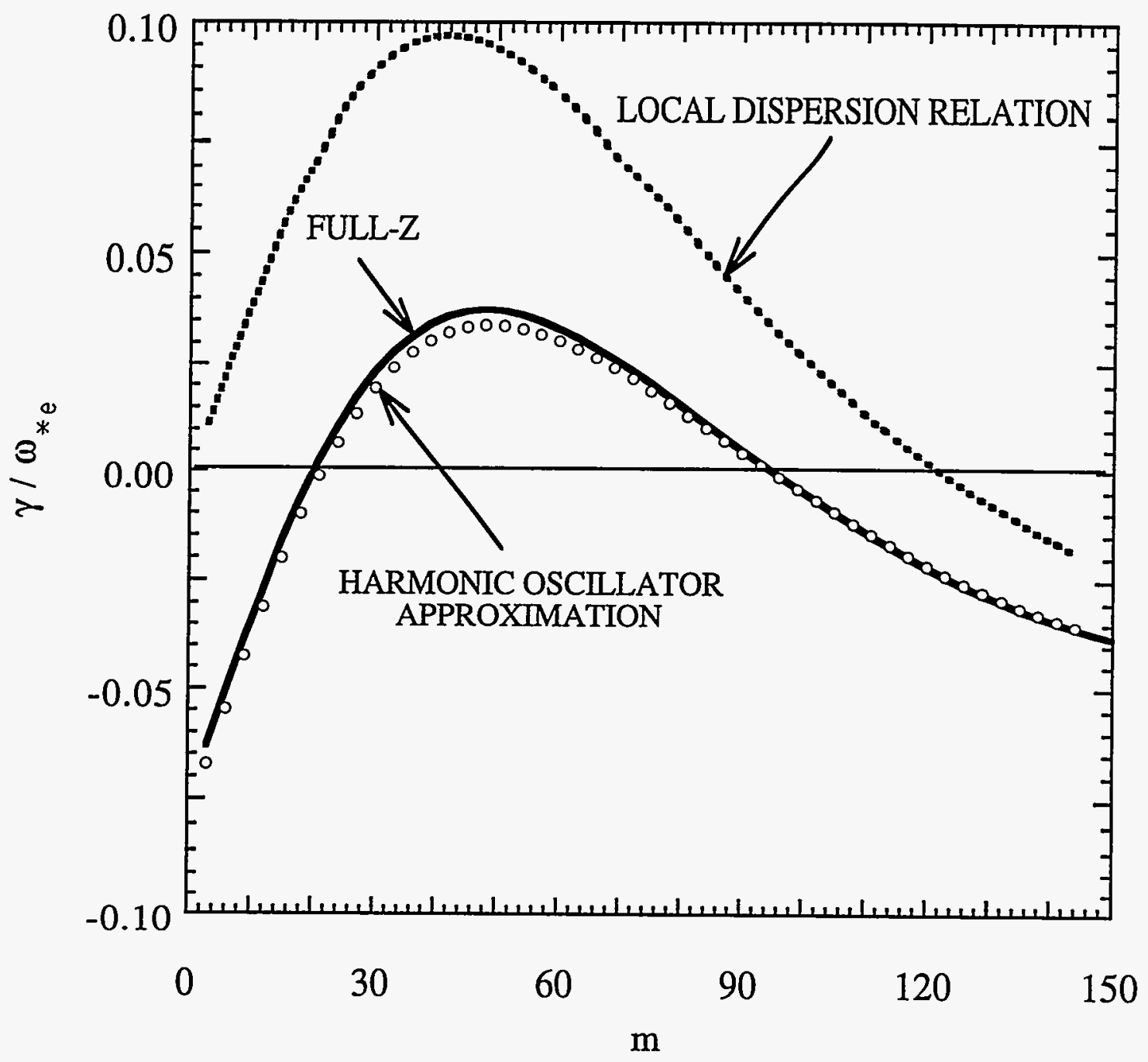

FIGURE 3a 


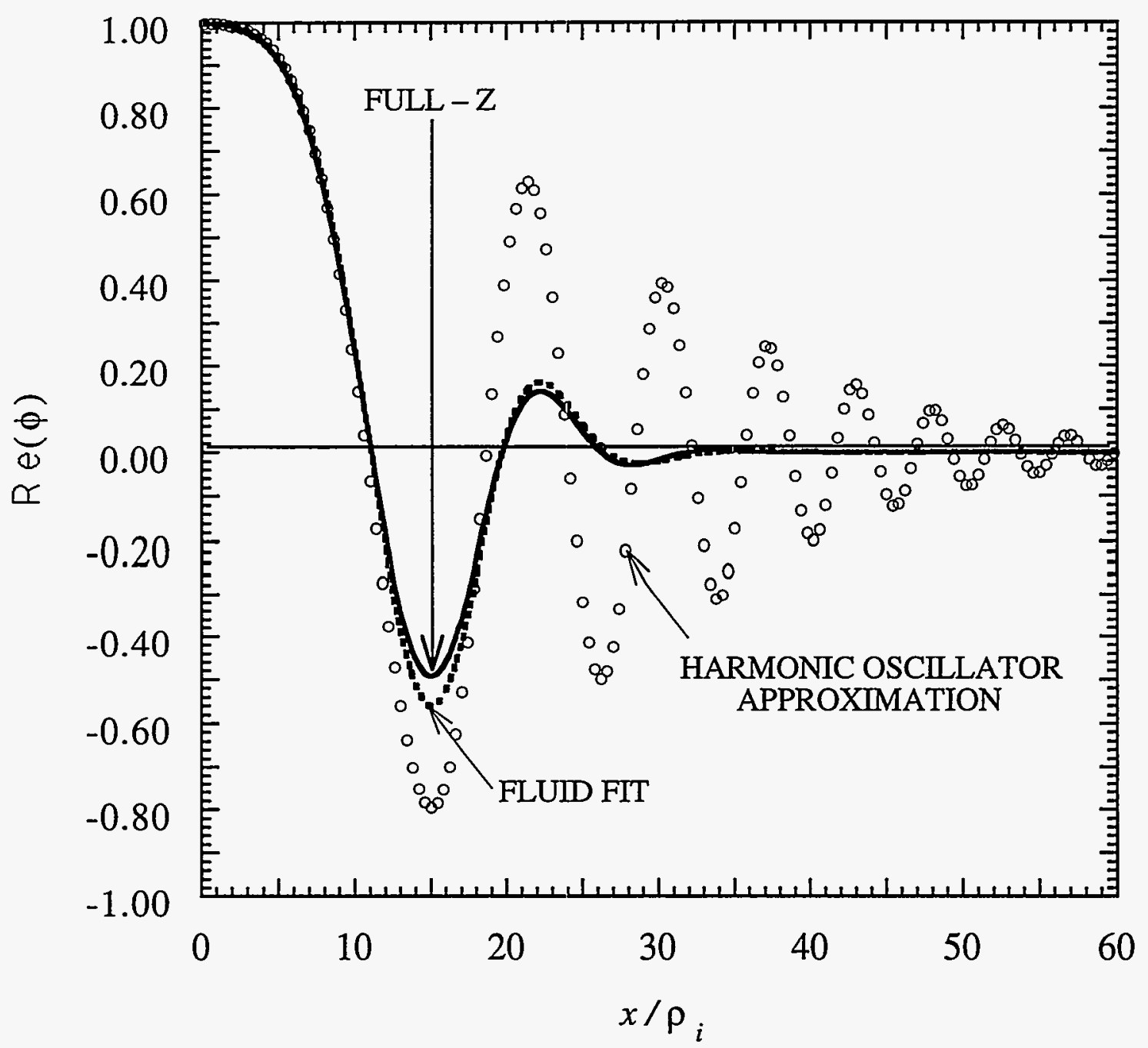

FIGURE 3b 


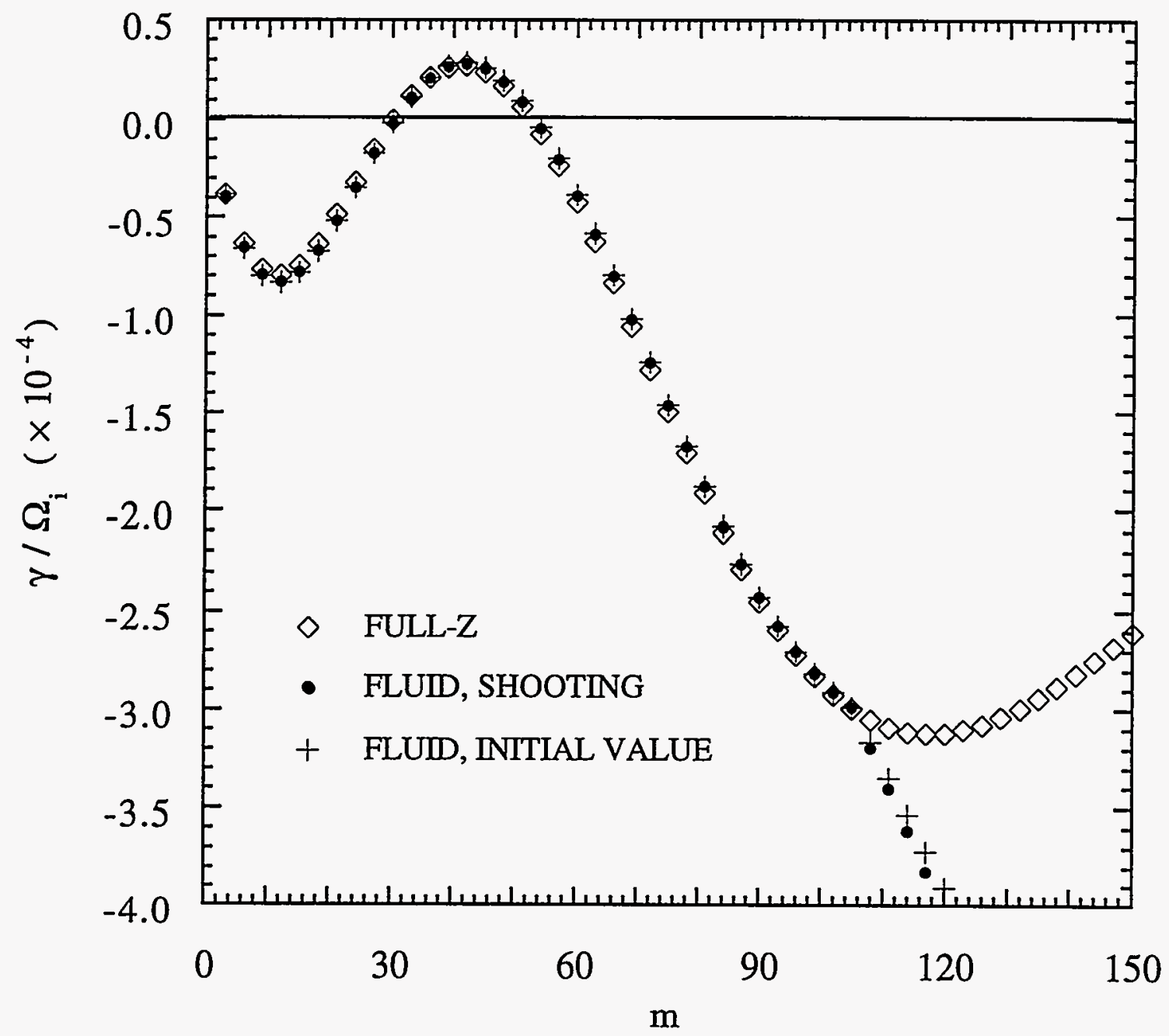

FIGURE 4a 


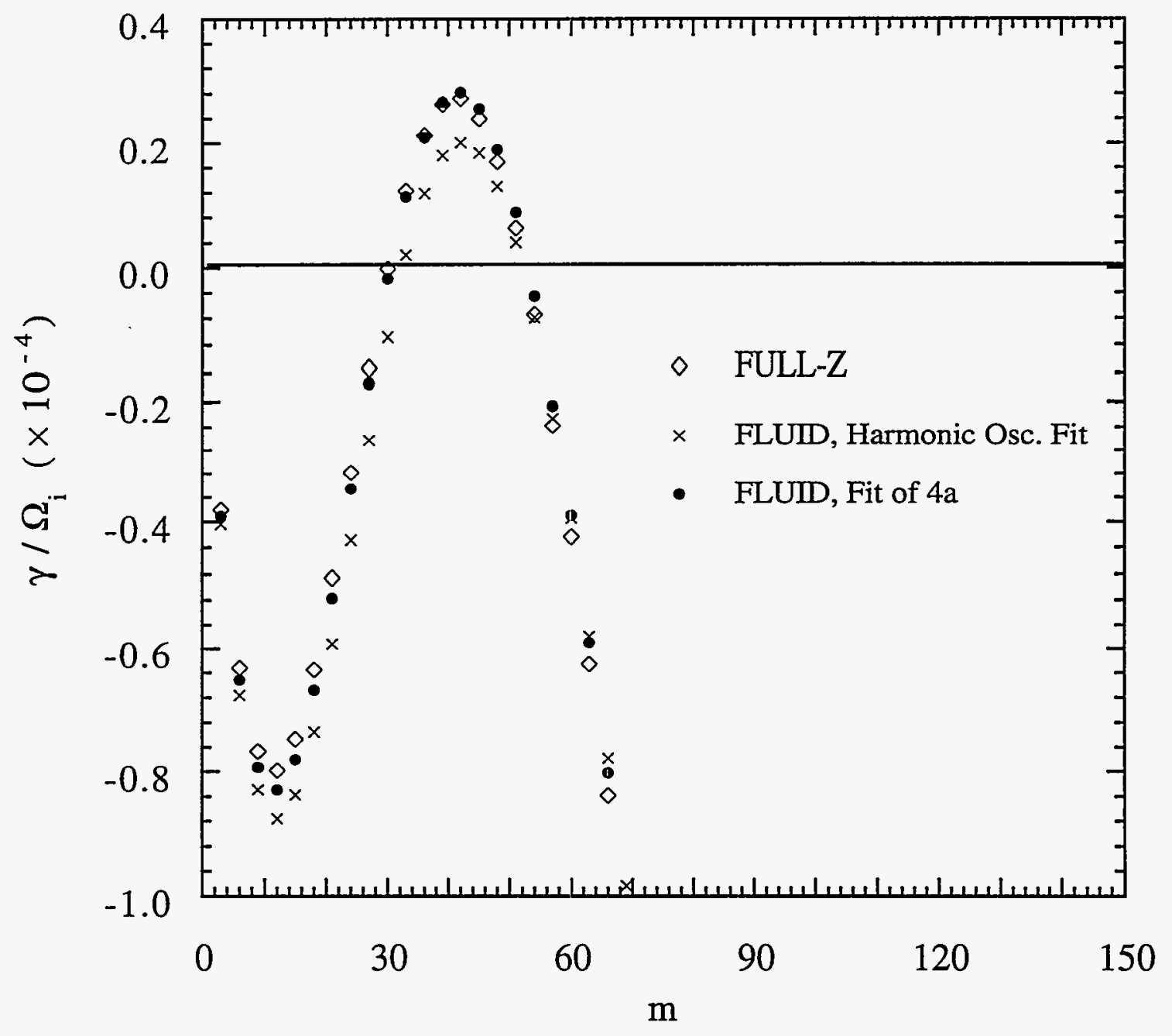

FIGURE 4b 


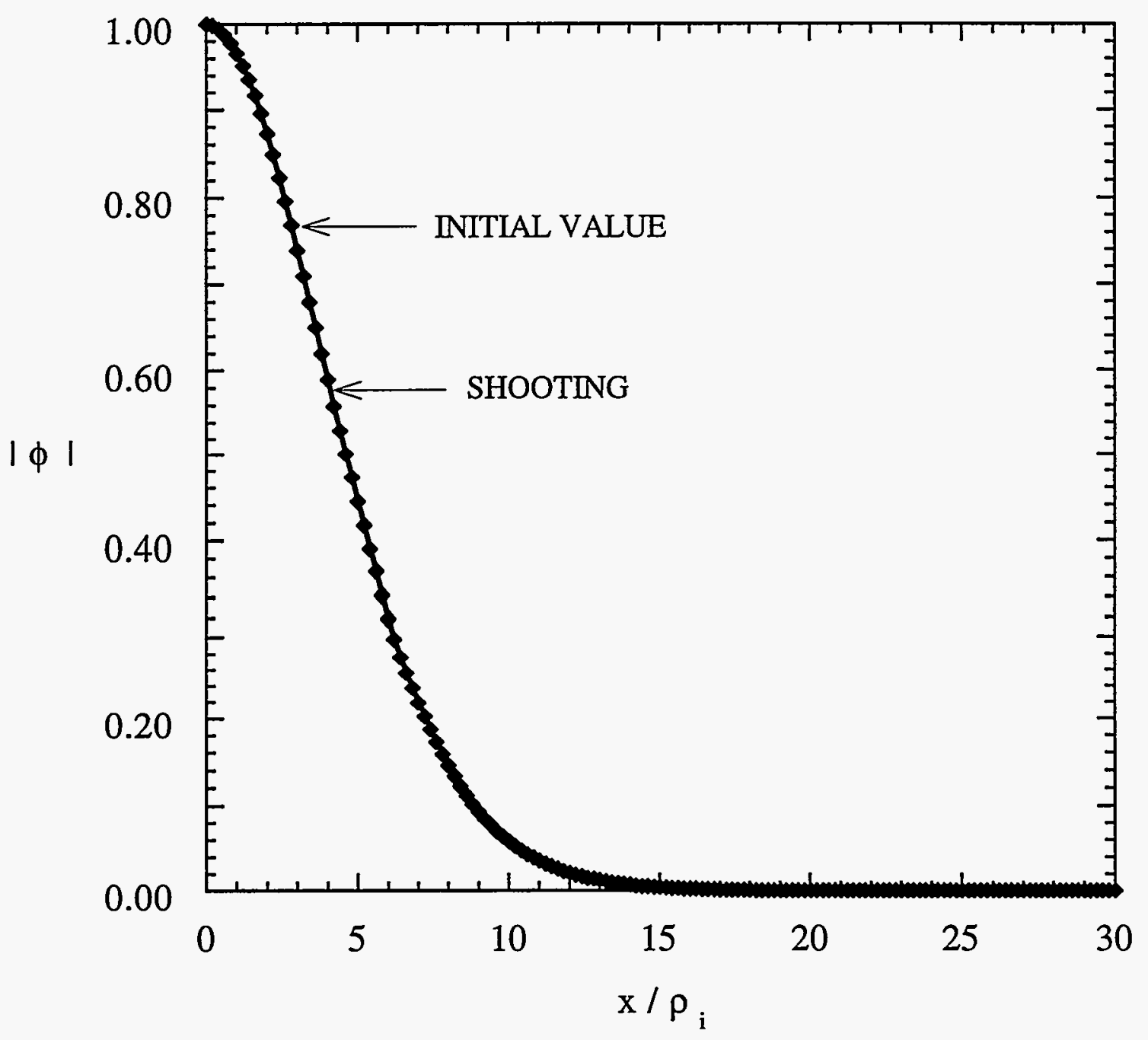

FIGURE 5 


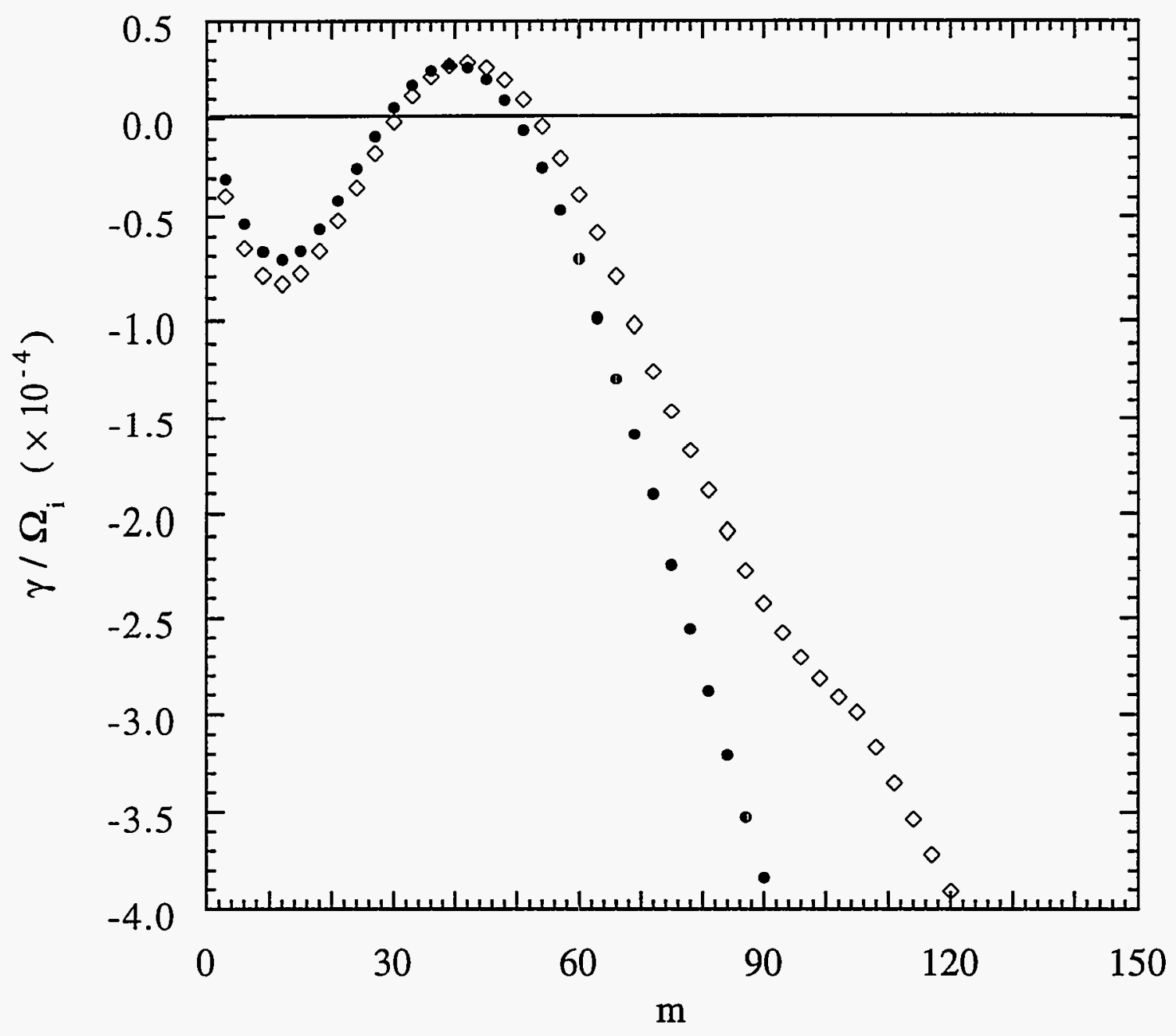

FIGURE 6 


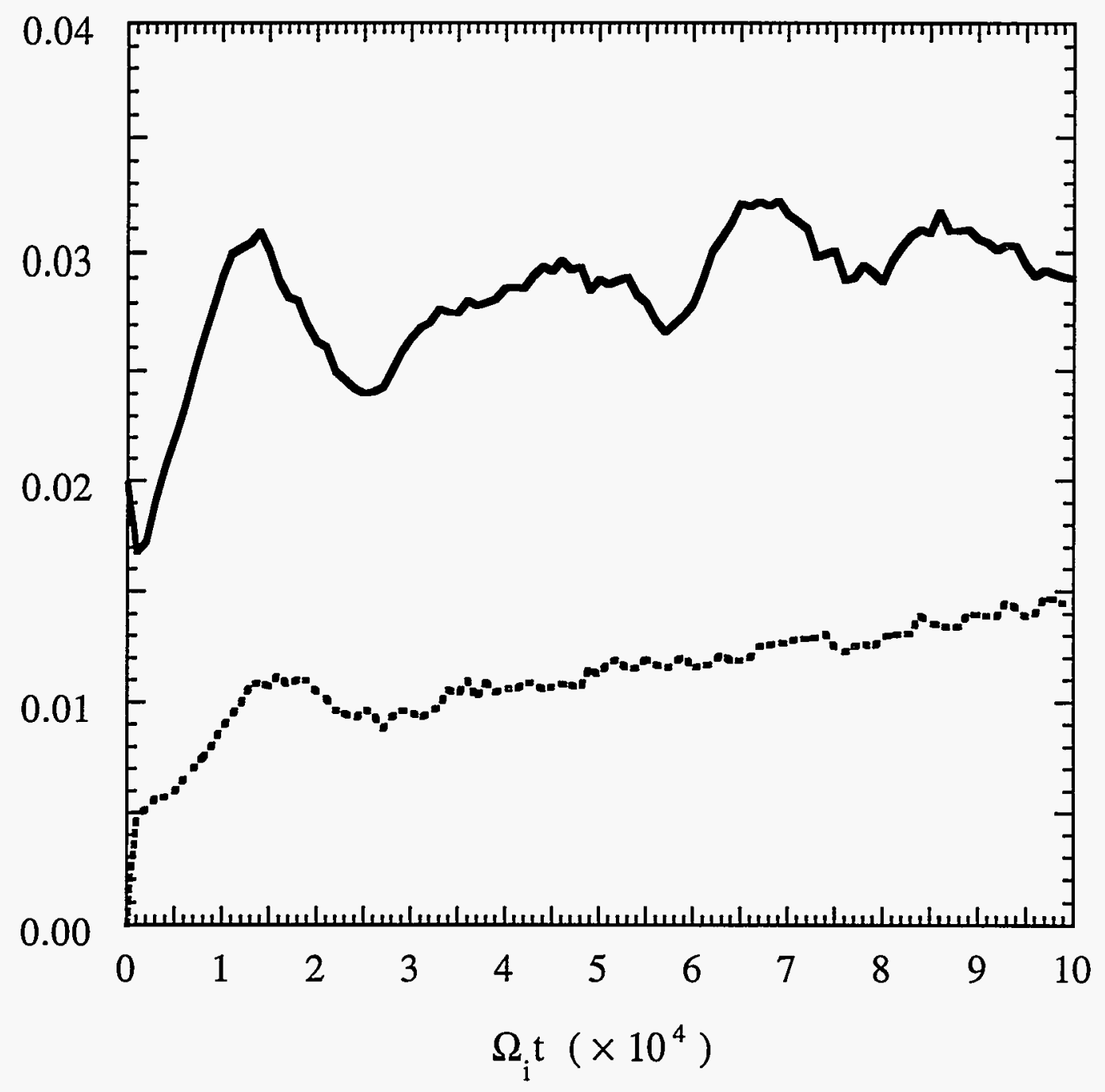

FIGURE 7 


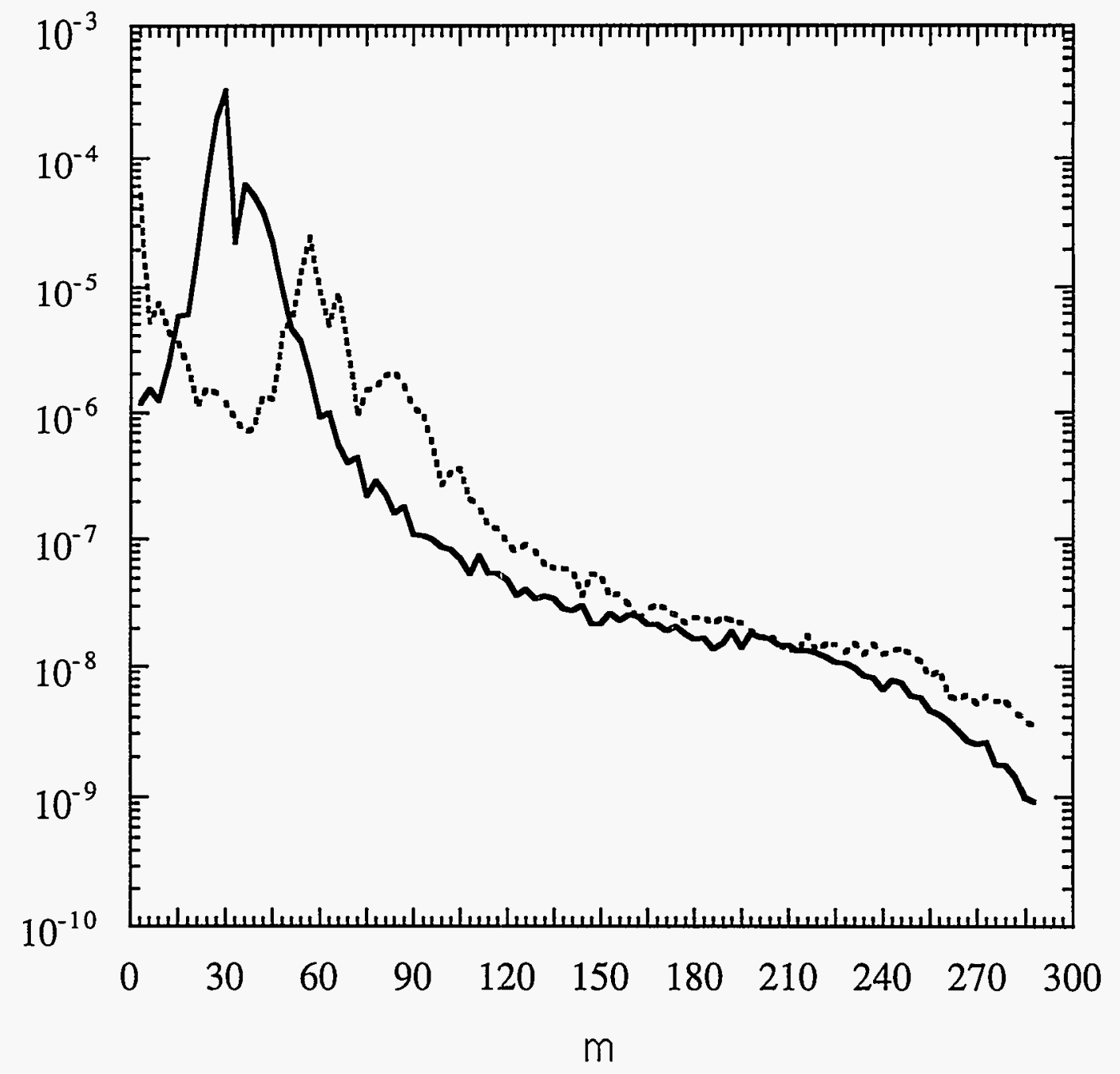

FIGURE 8 
ORNL/TM-13074

Dist. Category UC-427

\section{INTERNAL DISTRIBUTION}

1. Director, ORNL Fusion Energy Division

2. D. B. Batchelor

3. L. A. Berry

4. B. A. Carreras

5. H. E. Clark

6. R. J. Colchin

7. S. L. Milora

8-9. Laboratory Records Department

10. Laboratory Records, ORNL-RC

11-12. Central Research Library

13. Document Reference Section

14. Fusion Energy Division Library

15-16. ET/FE Division Publications Office

17. ORNL Patent Office

18. M. D. Carter

19. L. A. Charlton
20. K. R. Chen

21-51. J. W. Cobb

52. S. P. Hirshman

53. D. J. Hoffman

54. W. A. Houlberg

55. E. F. Jaeger

56. J. N. Leboeuf

57. V. E. Lynch

58. P. K. Mioduszewski

59. D. E. Newman

60. D. A. Spong

61. D. C. Stallings

62. C. Y. Wang

63. J. H. Whealton

64. J. C. Whitson

65. J. J. Yugo

\section{EXTERNAL DISTRIBUTION}

66. Office of the Assistant Manager for Energy Research and Development, Department of Energy, Oak Ridge Operations, P.O. Box 2000, Oak Ridge, TN 37831

67. N. A. Davies, Director, Office of Fusion Energy, Office of Energy Research, ER-50

Germantown, U.S. Department of Energy, Washington, DC 20545

68. M. Roberts, International Programs, Office of Fusion Energy, Office of Energy

Research, ER-52 Germantown, U.S. Department of Energy, Washington, DC 20545

69. D. E. Baldwin, Lawrence Livermore National Laboratory, P.O. Box 5511, Livermore, CA 94550

70. R. W. Conn, Mechanical, Aerospace, and Nuclear Engineering Department, 6291 Boelter Hall, University of California, Los Angeles, CA 90024-1597

71. P. C. Liewer, MS 138-208, Jet Propulsion Laboratory, 4800 Oak Grove Drive, Pasadena, CA 91109

72. D. Sigmar, Plasma Fusion Center, Massachusetts Institute of Technology, 167 Albany St., NW16-288, Cambridge, MA 02139

73. K. I. Thomassen, L-637, Lawrence Livermore National Laboratory, P.O. Box 5511, Livermore, CA 94550

74. J. D. Callen, Department of Nuclear Engineering, University of Wisconsin, Madison, WI 53706-1687

75. S. O. Dean, Fusion Power Associates, Inc., 2 Professional Drive, Suite 248, Gaithersburg, MD 20879

76. H. K. Forsen, Bechtel Group, Inc., Research Engineering, P.O. Box 3965, San Francisco, CA 94119

77. R. W. Gould, Department of Applied Physics, California Institute of Technology, Pasadena, CA 91125

78. R. A. Gross, Plasma Research Laboratory, Columbia University, New York, NY 10027 
79. R. J. Hawryluk, Plasma Physics Laboratory, Princeton University, P.O. Box 451, Princeton, NJ 08543

80. D. M. Meade, Plasma Physics Laboratory, Princeton University, P.O. Box 451, Princeton, NJ 08543

81. W. M. Stacey, School of Nuclear Engineering and Health Physics, Georgia Institute of Technology, Atlanta, GA 30332

82. D. Steiner, Nuclear Engineering Department, NES Building, Tibbetts Avenue, Rensselaer Polytechnic Institute, Troy, NY 12181

83. R. Varma, Physical Research Laboratory, Navrangpura, Ahmedabad 380009, India

84. Bibliothek, Max-Planck Institut für Plasmaphysik, Boltzmannstrasse 2, D-8046 Garching, Federal Republic of Germany

85. Bibliothek, Institut für Plasmaphysik, KFA Jülich GmbH, Postfach 1913, D-5170 Jülich, Federal Republic of Germany

86. Bibliothek, KfK Karlsruhe GmbH, Postfach 3640, D-7500 Karlsruhe 1, Federal Republic of Germany

87. Bibliotheque, Centre de Recherches en Physique des Plasmas, Ecole Polytechnique Fédérale de Lausanne, 21 Avenue des Bains, CH-1007 Lausanne, Switzerland

88. R. Aymar, CEN/Cadarache, Departement de Recherches sur la Fusion Contrôlée, F-13108 Saint-Paul-lez-Durance Cedex, France

89. Bibliothèque, CEN/Cadarache, F-13108 Saint-Paul-lez-Durance Cedex, France

90. Library, JET Joint Undertaking, Abingdon, Oxfordshire OX14 3EA, England

91. Library, FOM-Instituut voor Plasmafysica, Rijnhuizen, Edisonbaan 14, 3439 MN Nieuwegein, The Netherlands

92. Library, National Institute for Fusion Science, Chikusa-ku, Nagoya 464-01, Japan

93. Library, International Centre for Theoretical Physics, P.O. Box 586, I-34100 Trieste, Italy

94. Library, Centro Richerche Energia Frascati, C.P. 65, I-00044 Frascati (Roma), Italy

95. Library, Plasma Physics Laboratory, Kyoto University, Gokasho, Uji, Kyoto 611, Japan

96. Plasma Research Laboratory, Australian National University, P.O. Box 4, Canberra, A.C.T. 2601, Australia

97. Library, Japan Atomic Energy Research Institute, Naka Fusion Research Establishment, 801-1 Mukoyama, Naka-machi, Naka-gun, Ibaraki-ken, Japan

98. G. A. Eliseev, I. V. Kurchatov Institute of Atomic Energy, P.O. Box 3402, 123182 Moscow, Russia

99. V. A. Glukhikh, Scientific-Research Institute of Electro-Physical Apparatus, 188631 St. Petersburg, Russia

100. I. Shpigel, Institute of General Physics, U.S.S.R. Academy of Sciences, Ulitsa Vavilova 38, Moscow, Russia

101. D. D. Ryutov, Institute of Nuclear Physics, Siberian Academy of Sciences, Sovetskaya St. 5, 630090 Novosibirsk, Russia

102. O. Pavlichenko, Kharkov Physical-Technical Institute, Academical St. 1, 310108 Kharkov, Ukraine

103. Deputy Director, Southwestern Institute of Physics, P.O. Box 15, Leshan, Sichuan, China (PRC)

104. Director, The Institute of Plasma Physics, P.O. Box 1126, Hefei, Anhui, China (PRC)

105. R. H. McKnight, Experimental Plasma Research Branch, Division of Development and Technology, Office of Fusion Energy, Office of Energy Research, ER-542 Germantown, U.S. Department of Energy, Washington, DC 20545

106. E. Oktay, Division of Confinement Systems, Office of Fusion Energy, Office of Energy Research, ER-55 Germantown, U.S. Department of Energy, Washington, DC 20545

107. W. Sadowski, Fusion Theory and Computer Services Branch, Division of Applied Plasma Physics, Office of Fusion Energy, Office of Energy Research, ER-541 Germantown, U.S. Department of Energy, Washington, DC 20545

108. R. E. Mickens, Atlanta University, Department of Physics, Atlanta, GA 30314 
109. M. N. Rosenbluth, University of California at San Diego, La Jolla, CA 92037

110. D. Schnack, SAIC, 10260 Campus Point Drive, San Diego, CA 92121

111. Duk-In Choi, Department of Physics, Korea Advanced Institute of Science and Technology, P.O. Box 150, Chong Ryang-Ri, Seoul, Korea

112. Library of Physics Department, University of Ioannina, Ioannina, Greece

113. C. De Palo, Library, Associazione EURATOM-ENEA sulla Fusione, CP 65, I-00044 Frascati (Roma), Italy

114. Laboratorio Associado de Plasma, Instituto Nacional de Pesquisas Espaciais, Caixa Postal 515, 122201, Sao Jose dos Campos, SP, Brazil

115. Theory Department Read File, c/o D. W. Ross, University of Texas, Institute for Fusion Studies, Austin, TX 78712

116. Theory Department Read File, c/o R. Parker, Director, Plasma Fusion Center, NW 16-202, Massachusetts Institute of Technology, Cambridge, MA 02139

117. Theory Department Read File, c/o R. White, Plasma Physics Laboratory, Princeton University, P.O. Box 451, Princeton, NJ 08543

118. Theory Department Read File, c/o L. Kovrizhnykh, Lebedev Institute of Physics, Academy of Sciences, 53 Leninsky Prospect, 117924 Moscow, Russia

119. Theory Department Read File, c/o B. B. Kadomtsev, I. V. Kurchatov Institute of Atomic Energy, P.O. Box 3402, 123182 Moscow, Russia

120. Theory Department Read File, c/o T. Kamimura, National Institute for Fusion Studies, Nagoya 464, Japan

121. Theory Department Read File, c/o E. Maschke, Departemente de Recherches sur la Fusion Controlee, CEN/Cadarache, F-13108 Saint-Paul-lez Durance, France

122. Theory Department Read File, c/o D. Düchs, JET Joint Undertaking, Abingdon, Oxfordshire OX14 3EA, United Kingdom

123. Theory Department Read File, c/o R. Briscoe, Culham Laboratory, Abingdon, Oxfordshire OX14 3DB, United Kingdom

124. Theory Department Read File, c/o D. Biskamp, Max-Planck-Institut fur Plasmaphysik, Boltzmannstrasse 2, D-8046 Garching, Federal Republic of Germany

125. Theory Department Read File, c/o T. Takeda, Japan Atomic Energy Research Institute, Tokai Fusion Research Establishment, Tokai-mura, Naka-gun, Ibaraki-ken, Japan

126. Theory Department Read File, c/o J. Greene, General Atomics, P.O. Box 85608, San Diego, CA 92186

127. Theory Department Read File, c/o R. Cohen, Lawrence Livermore National Laboratory, P.O. Box 5511, Livermore, CA 94550

128. Theory Department Read File, c/o R. Gerwin, CTR Division, Los Alamos National Laboratory, P.O. Box 1663, Los Alamos, NM 87545

129. R. Aamodt, Lodestar Research Corporation, 2400 Central Avenue, Boulder, CO 80301

130. K. Appert, Centre de Recherches en Physique des Plasmas, Ecole Polytechnique Fédérale de Lausanne, 21 Avenue des Bains, CH-1007 Lausanne, Switzerland

131. M. Ballico, Max-Planck Institut für Plasmaphysik, Boltzmannstrasse 2, D-8046 Garching, Federal Republic of Germany

132. E. Barbato, Associazione EURATOM-ENEA sulla Fusione, Centro Richerche Energia Frascati, C.P. 65, I-00044 Frascati (Roma), Italy

133. A. Bécoulet, Association EURATOM-CEA sur la Fusion Controlée, Centre d'Etudes Nucléaires de Cadarache, B. P. No. 1, F-13108 Saint Paul lez Durances, France

134. S. Bernabei, Plasma Physics Laboratory, Princeton University, P.O. Box 451, Princeton, NJ 08543

135. A. Bers, Plasma Fusion Center, Massachusetts Institute of Technology, 38-260, Cambridge, MA 02139

136. V. Bhatnagar, JET Joint Undertaking, Abingdon, Oxfordshire OX14 3EA, England

137. P. T. Bonoli, Plasma Fusion Center, Massachusetts Institute of Technology, 175 Albany St., NW17-115, Cambridge, MA 02139. 
138. M. Brambilla, Max-Planck Institut für Plasmaphysik, Boltzmannstrasse 2, D-8046 Garching, Federal Republic of Germany

139. Dr. Vincent Chan, General Atomics, P. O. Box 85608, San Diego, CA 92186

140. D. Ehst, Building 205, Argonne National Laboratory, 9700 S. Cass Avenue, Argonne, IL 60538

141. D. Faulconer, Laboratoire de Physique des Plasmas, EURATOM-Etat Belge, Ecole Royale Militaire, Avenue de la Renaissance 30, 1040 Brussels, Belgium

142. D. J. Gambier, JET Joint Undertaking, Abingdon, Oxfordshire OX14 3EA, England

143. S. N. Golovato, Plasma Fusion Center, Massachusetts Institute of Technology, 175 Albany Street, NW17-113, Cambridge, MA 02139

144. W. Ho, SAIC, 10260 Campus Point Drive, San Diego, CA 92121

145. J. Hosea, Plasma Physics Laboratory, Princeton University, P.O. Box 451, Princeton, NJ 08543

146. Kwok Ko, Stanford Linear Accelerator Center, P.O. Box 4349, Stanford, CA 94309

147. C. C. Petty, General Atomics, P.O. Box 85608, San Diego, CA 92186

148. C. K. Phillips, Plasma Physics Laboratory, Princeton University, P.O. Box 451, Princeton, NJ 08543

149. M. Porkolab, Plasma Fusion Center, Massachusetts Institute of Technology, 175 Albany Street, NW17-119, Cambrid.ge, MA 02139

150. R. Prater, General Atomics, P.O. Box 85608, San Diego, CA 92186

151. J. Scharer, Electrical and Computer Engineering Department, 1500 Johnson Drive, University of Wisconsin, Madison, WI 53706

152. P. E. Vandenplas, Laboratoire de Physique des Plasmas, EURATOM-Etat Belge, Ecole Royale Militaire, Avenue de la Renaissance 30, 1040 Brussels, Belgium

153. H. Weitzner, Courant Institute for Mathematical Sciences, New York University, 251 Mercer Street, New York, NY 10001

154. J. R. Wilson, Plasma Physics Laboratory, Princeton University, P.O. Box 451, Princeton, NJ 08543

155-202. Given distribution according to OSTI-4500, Magnetic Fusion Energy (Category Distribution UC-427, Theoretical Plasma Physics) 